\title{
A SHORT COURSE ON GEOMETRIC MOTIVIC INTEGRATION
}

\author{
MANUEL BLICKLE
}

\begin{abstract}
These notes grew out of the authors effort to understand the theory of motivic integration. They give a short but thorough introduction to the flavor of motivic integration which nowadays goes by the name of geometric motivic integration. Motivic integration was introduced by Kontsevich and the foundations were worked out by Denef, Loeser, Batyrev and Looijenga. We focus on the smooth complex case and present the theory as self contained as possible. As an illustration we give some applications to birational geometry which originated in the work of Mustaţă.
\end{abstract}

\section{Contents}

1. The invention of motivic integration. 2

2. Geometric motivic integration 4

2.1. The value ring of the motivic measure 5

2.2. The arc space $\mathcal{J}_{\infty}(X) \quad 7$

2.3. An algebra of measurable sets 9

2.4. The measurable function associated to a subscheme 10

2.5. Definition and computation of the motivic integral 12

3. The transformation rule 14

3.1. Images of cylinders under birational maps. 16

3.2. Proof of transformation rule using Weak Factorization 19

4. Brief outline of a formal setup for the motivic measure. 20

4.1. Properties of the motivic measure 21

4.2. Motivic integration on singular varieties 24

5. Birational invariants via motivic integration 25

5.1. Notation from birational geometry 25

5.2. Proof of threshold formula 27

5.3. Bounds for the log canonical threshold 31

5.4. Inversion of adjunction 32

5.5. Geometry of arc spaces without explicit motivic integration. 34

Appendix A. An elementary proof of the Transformation rule. 37

A.1. The relative canonical divisor and differentials 37

A.2. Proof of Theorem 3.3 38

References $\quad 41$

Date: 28. July, 2005. 


\section{The InVEntion OF MOtiviC INTEGRATION.}

Motivic integration was introduced by Kontsevich [31] to prove the following result conjectured by Batyrev: Let

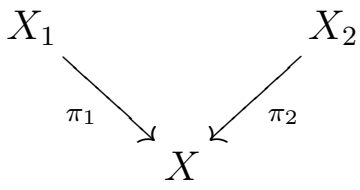

be two crepant resolutions of the singularities of $X$, which itself is a complex projective Calabi-Yau ${ }^{1}$ variety with at worst canonical Gorenstein singularities. Crepant (as in non discrepant) means that the pullback of the canonical divisor class on $X$ is the canonical divisor class on $X_{i}$, i.e. the discrepancy divisor $E_{i}=K_{X_{i}}-\pi_{i}^{*} K_{X}$ is numerically equivalent to zero. In this situation Batyrev showed, using $p$-adic integration, that $X_{1}$ and $X_{2}$ have the same betti numbers $h_{i}=\operatorname{dim} H^{i}(\ldots, \mathbb{C})$. This lead Kontsevich to invent motivic integration to show that $X_{1}$ and $X_{2}$ even have the same Hodge numbers $h^{i, j}=\operatorname{dim} H^{i}\left(\ldots, \Omega^{j}\right)$.

This problem was motivated by the topological mirror symmetry test of string theory which asserts that if $X$ and $X^{*}$ are a mirror pair ${ }^{2}$ of smooth Calabi-Yau varieties then they have mirrored Hodge numbers

$$
h^{i, j}(X)=h^{n-i, j}\left(X^{*}\right)
$$

As the mirror of a smooth Calabi-Yau might be singular, one cannot restrict to the smooth case and the equality of Hodge numbers actually fails in this case. Therefore Batyrev suggested, inspired by string theory, that one should look instead at the Hodge numbers of a crepant resolution, if such exists ${ }^{3}$. The independence of these numbers from the chosen crepant resolution is Kontsevich's result. This makes the stringy Hodge numbers $h_{s t}^{i, j}(X)$ of $X$, defined as $h^{i, j}\left(X^{\prime}\right)$ for a crepant resolution $X^{\prime}$ of $X$, well defined. This leads to a modified mirror symmetry conjecture, asserting that the stringy Hodge numbers of a mirror pair are equal [3].

Batyrev's conjecture is now Kontsevich's theorem and the simplest form to phrase it might be:

\footnotetext{
${ }^{1}$ Usually, a normal projective variety $X$ of dimension $n$ is called Calabi-Yau if the canonical divisor $K_{X}$ is trivial and $H^{i}\left(X, \mathcal{O}_{X}\right)=0$ for $0<i<n$. This last condition on the cohomology vanishing is not necessary for the statements below. In the context of mirror symmetry it seems customary to drop this last condition and call $X$ Calabi-Yau as soon as $K_{X}=0$ (and the singularities are mild), see [2].

${ }^{2}$ To explain what a mirror pair is in a useful manner lies beyond my abilities. For our purpose one can think of a mirror pair (somewhat tautologically) as being a pair that passes the topological mirror symmetry test. Another achievement of Batyrev [3] was to explicitly construct the mirror to a mildly singular (toric) Calabi-Yau variety.

${ }^{3}$ Calabi-Yau varieties do not always have crepant resolutions. I think one of Batyrev's papers discusses this.
} 
Theorem 1.1 (Kontsevich). Birationally equivalent smooth Calabi-Yau varieties have the same Hodge numbers. ${ }^{4}$

Proof. The idea now is to assign to any variety a volume in a suitable ring $\hat{\mathcal{M}}_{k}$ such that the information about the Hodge numbers is retained. The following diagram illustrates the construction of $\hat{\mathcal{M}}_{k}$ :

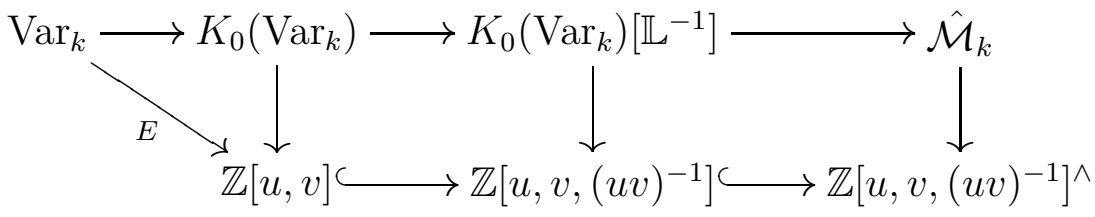

The diagonal map is the (compactly supported) Hodge characteristic, which on a smooth projective variety $X$ is given by $E(X)=\sum(-1)^{i} \operatorname{dim} H^{i}\left(X, \Omega_{X}^{j}\right) u^{i} v^{j}$. In general it is defined via mixed Hodge structures ${ }^{5}[8,9,10]$, satisfies $E(X \times Y)=E(X) E(Y)$ for all varieties $X, Y$ and has the property that for $Y \subseteq X$ a closed $k$-subvariety one has $E(X)=E(Y)+E(X-Y)$. Therefore the Hodge characteristic factors through the naive Grothendieck ring $K_{0}\left(\operatorname{Var}_{k}\right)$ which is the universal object with the latter property. ${ }^{6}$ This explains the left triangle of the diagram.

The bottom row of the diagram is the composition of a localization (inverting $u v$ ) and a completion with respect to negative degree. $\mathcal{M}_{k}$ is constructed analogously, by first inverting $\mathbb{L}^{-1}=\left[\mathbb{A}_{k}^{1}\right]$ (a pre-image of $u v$ ) and then completing appropriately (negative dimension). Whereas the bottom maps are injective (easy exercise), the map $K_{0}\left(\operatorname{Var}_{k}\right) \rightarrow \hat{\mathcal{M}}_{k}$ is most likely not injective. The need to work with $\hat{\mathcal{M}}_{k}$ instead of $K_{0}\left(\operatorname{Var}_{k}\right)$ arises in the setup of the integration theory an will become clear later. ${ }^{7}$

Clearly, by construction it is now enough to show that birationally equivalent CalabiYau varieties have the same volume, i.e. the same class in $\hat{\mathcal{M}}_{k}$. This is achieved via the all important birational transformation rule of motivic integration. Roughly it asserts that for a proper birational map $\pi: Y \rightarrow X$ the class $[X] \in \hat{\mathcal{M}}_{k}$ is an expression in $Y$ and $K_{Y / X}$ only:

$$
[X]=\int_{Y} \mathbb{L}^{-\operatorname{ord}_{K_{Y / X}}}
$$

\footnotetext{
${ }^{4}$ There is now a proof by Ito [28] of this result using $p$-adic integration, thus continuing the ideas of Batyrev who proved the result for Betti numbers using this technique. Furthermore the recent weak factorization theorem of Włodarczyk [1] allows for a proof avoiding integration of any sort.

${ }^{5}$ Recently, Bittner [4] gave an alternative construction of the compactly supported Hodge characteristic. She uses the weak factorization theorem of Włodarczyk [1] to reduce the definition of $E$ to the case of $X$ smooth and projective, where it is as given above.

${ }^{6} K_{0}\left(\operatorname{Var}_{k}\right)$ is the free abelian group on the isomorphism classes $[X]$ of $k$-varieties subject to the relations $[X]=[X-Y]+[Y]$ for $Y$ a closed subvariety of $X$. The product is given by $[X][Y]=\left[X \times_{k} Y\right]$. The symbol $\mathbb{L}$ denotes the class of the affine line $\left[\mathbb{A}_{k}^{1}\right]$.

${ }^{7}$ In fact, recent results of F. Loeser and R. Cluckers [6], and J. Sebag [41] indicate that the full completion may not be necessary, and all the volumes of measurable sets are contained in a subring of $\hat{\mathcal{M}}_{k}$ that can be constructed explicitly.
} 
To finish off the proof let $X_{1}$ and $X_{2}$ be birationally equivalent Calabi-Yau varieties. We resolve the birational map to a Hironaka hut:

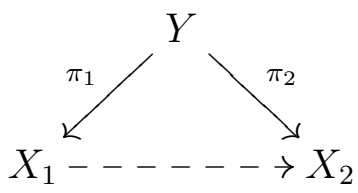

By the Calabi-Yau assumption we have $K_{X_{i}} \equiv 0$ and therefore $K_{Y / X_{i}} \equiv K_{Y}-$ $\pi_{i}^{*} K_{X_{i}} \equiv K_{Y}$. Hence the divisors $K_{Y / X_{1}}$ and $K_{Y / X_{2}}$ are numerically equivalent. This numerical equivalence implies in fact an equality of divisors $K_{X / X_{1}}=K_{X / X_{2}}$ since, again by the Calabi-Yau assumption, $\operatorname{dim} H^{0}\left(X, K_{Y}\right)=\operatorname{dim} H^{0}\left(X_{i}, \mathcal{O}_{X_{i}}\right)=1 .^{8}$ By the transformation rule, $\left[X_{1}\right]$ is an expression depending only on $Y$ and $K_{X / X_{1}}=K_{X / X_{2}}$. The same is true for $\left[X_{2}\right]$ and thus we have $\left[X_{1}\right]=\left[X_{2}\right]$ as desired.

These notes were started during a working seminar at MSRI during the year of 2003 and took shape in the course of the past 2 years while I was giving introductory lectures on the subject. They have taken me way too much time to finish and would not have been finished at all if it weren't for the encouragement of many people: Thanks goes to all the participants of the seminar on motivic integration at MSRI (2002/2003), of the Schwerpunkt Junioren Tagung in Bayreuth (2003) and the patient listeners of the mini-courses at KTH, Stockholm (2003), the University of Helsinki (2004) and the Vigre graduate course in Salt Lake City (2005). Special thanks goes to Karen Smith for encouragement to start this project and to Julia Gordon for numerous comments, suggestions and careful reading.

\section{Geometric motivic integration}

We now assume that $k$ is algebraically closed and of characteristic zero. In fact, there is one point (see section 4.1) where we will assume that $k=\mathbb{C}$ in order to avoid some technicalities which arise if the field is not uncountable. Thus the reader may choose to replace $k$ by $\mathbb{C}$ whenever it is comforting. We stress that there are significant (though manageable) obstacles one has to overcome if one wants to (a) work with singular spaces or (b) with varieties defined over fields which are not uncountable or not algebraically closed. Or put differently: The theory develops naturally (for an algebraic geometer), and easily, in the smooth case over $\mathbb{C}$, as we hope to demonstrate below. In order to transfer this intuition to any other situation, nontivial results and extra care is necessary.

All the results in these notes appeared in the papers of Denef and Loeser, Batyrev and Looijenga. Our exposition is particularly influenced by Looijenga [33] and Batyrev [2]. Also Craw [7] was very helpful as a first reading. We also recommend the articles

\footnotetext{
${ }^{8}$ In general, the condition that $X_{1}$ and $X_{2}$ have a common resolution $Y$ such that $K_{Y / X_{1}}$ is numerically equivalent $K_{Y / X_{1}}$ is called $K$-equivalence. We showed above that two birational Calabi-Yau varieties are $K$-equivalent. For mildly singular $X_{i}$ (say canonical) it can be derived from the negativity lemma [30, Lemma 3.39] that $K$-equivalence implies actual equality of divisors $K_{Y / X_{1}}$ and $K_{Y / X_{1}}$. Hence the Calabi-Yau assumption was not essential to conclude this (but provides a simple argument).
} 
of Hales [25] and Veys [44], both explain the connection to p-adic integration in detail, which we do not discuss in these notes at all. The above mentioned references are also a great source to learn about the various different applications the theory had to date. We will discuss none of them except for certain applications to birational geometry.

We will now introduce the building blocks of the theory. These are:

(1) The value ring of the measure: $\hat{\mathcal{M}}_{k}$, a localized and completed Grothendieck ring.

(2) A domain of integration: $\mathcal{J}_{\infty}(X)$, the space of formal arcs over $X$.

(3) An algebra of measurable sets of $\mathcal{J}_{\infty}(X)$ and a measure: cylinders/stable sets and the virtual euler characteristic.

(4) An interesting class of measurable/integrable functions: Contact order of an arc along a divisor.

(5) A change of variables formula: Kontsevich's birational transformation rule.

These basic ingredients appear with variations in all versions of motivic integration (one could argue: of any theory of integration).

2.1. The value ring of the motivic measure. Here we already gravely depart from any previous (classical?) theory of integration since the values of our measure do not lie in $\mathbb{R}$. Instead they lie in a huge ring, constructed from the Grothendieck ring of varieties by a process of localization and completion. This ingenious choice is a key feature of the theory.

We start with the naive Grothendieck ring of the category of varieties over $k{ }^{9}$ This is the ring $K_{0}\left(\operatorname{Var}_{k}\right)$ generated by the isomorphism classes of all finite type $k$-varieties and with relation $[X]=[Y]+[X-Y]$ for a closed $k$-subvariety $Y \subseteq X$, that is such that the inclusion $Y \subseteq X$ is defined over $k$. The square brackets denote the image of $X$ in $K_{0}(\operatorname{Var} / k)$. The product structure is given by the fiber product, $[X] \cdot[Y]=$ $\left[X \times_{k} Y\right]\left(=\left[\left(X \times_{k} Y\right)_{\text {red }}\right]\right)$. The symbol $\mathbb{L}$ is reserved for the class of the affine line $\left[\mathbb{A}_{k}^{1}\right]$ and $1=1_{k}$ denotes Spec $k$. Thus, for example, $\left[\mathbb{P}^{n}\right]=\mathbb{L}^{n}+\mathbb{L}^{n-1}+\ldots+\mathbb{L}+1$.

Roughly speaking the map $X \mapsto[X]$ is robust with respect to chopping up $X$ into a disjoint union of locally closed subvarieties. ${ }^{10}$ By using a stratification of $X$ by smooth subvarieties, this shows that $K_{0}\left(\operatorname{Var}_{k}\right)$ is generated by the classes of smooth varieties. ${ }^{11}$ In a similar fashion one can assign to every constructible subset $C$ of $X$ a class $[C]$ by expressing $C$ as a combination of subvarieties.

\footnotetext{
${ }^{9}$ Alternatively, the Grothendieck ring of finite type schemes over $k$ leads to the same object because $X-X_{\text {red }}=\emptyset$. As Bjorn Poonen points out, the finite type assumption is crucial here. If one would allow non finite type schemes, $K_{0}\left(\operatorname{Var}_{k}\right)$ would be zero. For this let $Y$ be any $k$-scheme and let $X$ be an infinite disjoint union of copies of $Y$. Then $[X]+[Y]=[X]$ and therefore $[Y]=0$.

${ }^{10}$ This is elegantly illustrated in the article of Hales [25] which emphasizes precisely this point of $K_{0}\left(\operatorname{Var}_{k}\right)$ being a scissor group.

${ }^{11}$ In [4], Bittner shows that $K_{0}\left(\operatorname{Var}_{k}\right)$ is the abelian group generated by smooth projective varieties subject to a class of relations which arise from blowing up at a smooth center: If $Z$ is a smooth subvariety of $X$, then the relation is $[X]-[Z]=\left[\mathrm{Bl}_{Z} X\right]-[E]$, where $E$ is the exceptional divisor of the blowup.
} 
Exercise 2.1. Verify the claim in the last sentence. That is: show that the map $Y \mapsto[Y]$ for $Y$ a closed subvariety of $X$ naturally extends to the algebra of constructible subsets of $X$.

Exercise 2.2. Let $Y \rightarrow X$ be a piecewise trivial fibration with constant fiber $Z$. This means one can write $X=\bigsqcup X_{i}$ as a finite disjoint union of locally closed subsets $X_{i}$ such that over each $X_{i}$ one has $f^{-1} X_{i} \cong X_{i} \times Z$ and $f$ is given by the projection onto $X_{i}$. Show that in $K_{0}\left(\operatorname{Var}_{k}\right)$ one has $[Y]=[X] \cdot[Z]$.

There is a natural notion of dimension of an element of $K_{0}\left(\operatorname{Var}_{k}\right)$. We say that $\tau \in K_{0}\left(\operatorname{Var}_{k}\right)$ is $d$-dimensional if there is an expression in $K_{0}\left(\operatorname{Var}_{k}\right)$

$$
\tau=\sum a_{i}\left[X_{i}\right]
$$

with $a_{i} \in \mathbb{Z}$ and $k$-varieties $X_{i}$ of dimension $\leq d$, and if there is no expression like this with all $\operatorname{dim} X_{i} \leq d-1$. The dimension of the class of the empty variety is set to be $-\infty$. It is easy to verify (exercise!) that the map

$$
\operatorname{dim}: K_{0}\left(\operatorname{Var}_{k}\right) \rightarrow \mathbb{Z} \cup\{-\infty\}
$$

satisfies $\operatorname{dim}\left(\tau \cdot \tau^{\prime}\right) \leq \operatorname{dim} \tau+\operatorname{dim} \tau^{\prime}$ and $\operatorname{dim}\left(\tau+\tau^{\prime}\right) \leq \max \left\{\operatorname{dim} \tau, \operatorname{dim} \tau^{\prime}\right\}$ with equality in the latter if $\operatorname{dim} \tau \neq \operatorname{dim} \tau^{\prime}$.

The dimension can be extended to the localization $\mathcal{M}_{k} \stackrel{\text { def }}{=} K_{0}\left(\operatorname{Var}_{k}\right)\left[\mathbb{L}^{-1}\right]$ simply by demanding that $\mathbb{L}^{-1}$ has dimension -1 . To obtain the ring $\hat{\mathcal{M}}_{k}$ in which the desired measure will take values we further complete $\mathcal{M}_{k}$ with respect to the filtration induced by the dimension. ${ }^{12}$ The $n$th filtered subgroup is

$$
\mathcal{F}^{n}\left(\mathcal{M}_{k}\right)=\left\{\tau \in \mathcal{M}_{k} \mid \operatorname{dim} \tau \leq n\right\} .
$$

This gives us the following maps which will be the basis for constructing the sought after motivic measure:

$$
\operatorname{Var}_{k} \longrightarrow K_{0}\left(\operatorname{Var}_{k}\right) \stackrel{\text { invert } \mathbb{L}}{\longrightarrow} \mathcal{M}_{k} \stackrel{\wedge}{\longrightarrow} \hat{\mathcal{M}}_{k}
$$

\footnotetext{
${ }^{12}$ In Looijenga [33], this is called the virtual dimension. As described by Batyrev, composing the dimension $\operatorname{dim}: \mathcal{M}_{k} \longrightarrow \mathbb{Z} \cup\{-\infty\}$ with the exponential $\mathbb{Z} \subseteq \mathbb{R} \stackrel{\exp (-)}{\longrightarrow} \mathbb{R}_{+}$and by further defining $\emptyset \mapsto 0$ we get a map

$$
\delta_{k}: \mathcal{M}_{k} \longrightarrow \mathbb{R}_{+, 0}
$$
}

which is a non-archimedian norm. That means the following properties hold:

(1) $\delta_{k}(A)=0$ iff $A=0=[\emptyset]$ in $\mathcal{M}_{k}$.

(2) $\delta_{k}(A+B) \leq \max \left\{\delta_{k}(A), \delta_{k}(B)\right\}$

(3) $\delta_{k}(A \cdot B) \leq \delta_{k}(A) \cdot \delta_{k}(B)$

The ring $\hat{\mathcal{M}}_{k}$ is then the completion with respect to this norm, and therefore $\hat{\mathcal{M}}_{k}$ is complete in the sense that all Cauchy sequences uniquely converge. The condition (2) is stronger than the one used in the definition of an archimedian norm. This non-archimedian ingredient makes the notion of convergence of sums conveniently simple; a sum converges if and only if the sequence of summands converges to zero.

If there was an equality in condition (3) the norm would be called multiplicative. It is unknown whether $\delta$ is multiplicative on $\mathcal{M}_{k}$. However, Poonen [39] shows that $K_{0}\left(\operatorname{Var}_{k}\right)$ contains zero divisors, thus $\delta$ restricted to $K_{0}\left(\operatorname{Var}_{k}\right)$ is not multiplicative on $K_{0}\left(\operatorname{Var}_{k}\right)$. 
We will somewhat ambiguously denote the image of $X \in \operatorname{Var}_{k}$ in any of the rings to the right by $[X]$. It is important to point out here that it is unknown whether the completion map _ $\wedge$ is injective, i.e. whether its kernel, $\cap \mathcal{F}^{d}\left(K_{0}(\operatorname{Var} / k)\left[\mathbb{L}^{-1}\right]\right)$, is zero. It is also unknown whether the localization is injective. ${ }^{13}$

Exercise 2.3. Convergence of series in $\hat{\mathcal{M}}_{k}$ is rather easy. For this observe that a sequence of elements $\tau_{i} \in \mathcal{M}_{k}$ converges to zero in $\hat{\mathcal{M}}_{k}$ if and only if the dimensions $\operatorname{dim} \tau_{i}$ tend to $-\infty$ as $i$ approaches $\infty$. Show that a sum $\sum_{i=0}^{\infty} \tau_{i}$ converges if and only the sequence of summands converges to zero.

Exercise 2.4. Show that in $\hat{\mathcal{M}}_{k}$ the equality $\sum_{i=0}^{\infty} \mathbb{L}^{-k i}=\frac{1}{1-\mathbb{L}^{-k}}$ holds.

2.2. The arc space $\mathcal{J}_{\infty}(X)$. Arc spaces were first studied seriously by Nash [37] who conjectured a tight relationship between the geometry of the arc space and the singularities of $X$, see Ishii and Kollar [27] for a recent exposition of Nash's ideas in modern language. Recent work of Mustaţă [35] supports these predictions by showing that the arc spaces contain information about singularities, for example rational singularities of $X$ can be detected by the irreducibility of the jet schemes for complete intersections. In subsequent investigations he and his collaborators show that certain invariants of birational geometry, such as the log canonical threshold of a pair, for example, can be read off from the dimensions of certain components of the jet schemes, see $[36,15,17]$ and Section 5 where we will discuss some of these results in detail.

Let $X$ be a (smooth) scheme of finite type over $k$ of dimension $n$. An $m$-jet of $X$ is an order $m$ infinitesimal curve in $X$, i.e. it is a morphism

$$
\vartheta: \operatorname{Spec} k[t] / t^{m+1} \longrightarrow X .
$$

The set of all $m$-jets carries the structure of a scheme $\mathcal{J}_{m}(X)$, called the $m$ th jet scheme, or space of truncated arcs. It's characterizing property is that it is right adjoint to the functor $\_\times$Spec $k[t] / t^{m+1}$. In other words,

$$
\operatorname{Hom}\left(Z \times \operatorname{Spec} k[t] / t^{m+1}, X\right)=\operatorname{Hom}\left(Z, \mathcal{J}_{m}(X)\right)
$$

for all $k$-schemes $Z$, i.e. $\mathcal{J}_{m}(X)$ is the scheme which represents the contravariant functor $\operatorname{Hom}\left(\ldots \times \operatorname{Spec} k[t] / t^{m+1}, X\right) .{ }^{14}$ In particular this means that the $k$-valued points of $\mathcal{J}_{m}(X)$ are precisely the $k[t] / t^{m+1}$-valued points of $X$. The so called Weil restriction of scalars, i.e. the natural map $k[t] / t^{m+1} \longrightarrow k[t] / t^{m}$, induces a map $\pi_{m-1}^{m}: \mathcal{J}_{m}(X) \longrightarrow$ $\mathcal{J}_{m-1}(X)$ and composition gives a map $\pi^{m}: \mathcal{J}_{m}(X) \rightarrow \mathcal{J}_{0}(X)=X$. As upper indices are often cumbersome we define $\eta_{m}=\pi^{m}$ and $\varphi_{m}=\pi_{m-1}^{m}$.

\footnotetext{
${ }^{13}$ In [39] Poonen shows that $K_{0}(\operatorname{Var} / k)$ is not a domain in characteristic zero. It is expected though that the localization map is not injective and that $\mathcal{M}_{k}$ is a domain and that the completion map $\mathcal{M}_{k} \longrightarrow \hat{\mathcal{M}}_{k}$ is injective. But recently Naumann [38] found in his dissertation zero-divisors in $K_{0}\left(\operatorname{Var}_{k}\right)$ for $k$ a finite field and these are non-zero even after localizing at $\mathbb{L}$ - thus for a finite field $\mathcal{M}_{k}$ is not a domain. For infinite fields (e.g. $k$ algebraically closed) the above questions remain open.

${ }^{14}$ Representability of this functor was proved by Greenberg [19, 20]; another reference for this fact is $[5]$.
} 
Taking the inverse limit ${ }^{15}$ of the resulting system yields the definition of the infinite jet scheme, or the arc space

$$
\mathcal{J}_{\infty}(X)=\lim _{\longleftarrow} \mathcal{J}_{m}(X) .
$$

Its $k$-points are the limit of the $k$-valued points $\operatorname{Hom}\left(\operatorname{Spec} k[t] / t^{m+1}, X\right)$ of the jet spaces $\mathcal{J}_{m}(X)$. Therefore they correspond to the formal curves (or arcs) in $X$, that is to maps Spec $k \llbracket t \rrbracket \rightarrow X{ }^{16}$ There are also maps $\pi_{m}: \mathcal{J}_{\infty}(X) \longrightarrow \mathcal{J}_{m}(X)$ again induced by the truncation map $k \llbracket t \rrbracket \rightarrow k \llbracket t \rrbracket / t^{m+1}$. If there is danger of confusion we sometimes decorate the projections $\pi$ with the space. The following picture should help to remember the notation.

$$
\mathcal{J}_{\infty}(X) \stackrel{\pi_{a}}{\longrightarrow} \mathcal{J}_{a}(X) \stackrel{\pi_{b}^{a}}{\longrightarrow} \mathcal{J}_{b}(X) \stackrel{\pi^{b}}{\eta_{b}} X
$$

are the maps induced by the natural surjections

$$
k \llbracket t \rrbracket \longrightarrow k \llbracket t \rrbracket / t^{a+1} \longrightarrow k \llbracket t \rrbracket / t^{b+1} \longrightarrow k
$$

Example 2.1. Let $X=\operatorname{Spec} k\left[x_{1}, \ldots, x_{n}\right]=\mathbb{A}^{n}$. Then, on the level of $k$-points one has

$$
\mathcal{J}_{m}(X)=\left\{\vartheta: k\left[x_{1}, \ldots, x_{n}\right] \rightarrow k \llbracket t \rrbracket / t^{m+1}\right\}
$$

Such a map $\vartheta$ is determined by its values on the $x_{i}$ 's, i.e. it is determined by the coefficients of $\vartheta\left(x_{i}\right)=\sum_{j=0}^{m} \vartheta_{i}^{(j)} t^{j}$. Conversely, any choice of coefficients $\vartheta_{i}^{(j)}$ determines a point in $\mathcal{J}_{m}\left(\mathbb{A}^{n}\right)$. Choosing coordinates $x_{i}^{(j)}$ of $\mathcal{J}_{m}(X)$ with $x_{i}^{(j)}(\vartheta)=\vartheta_{i}^{(j)}$ we see that

$$
\mathcal{J}_{m}(X) \cong \operatorname{Spec} k\left[x_{1}^{(0)}, \ldots, x_{n}^{(0)}, \ldots \ldots, x_{1}^{(m)}, \ldots, x_{n}^{(m)}\right] \cong \mathbb{A}^{n(m+1)} .
$$

Furthermore observe that, somewhat intuitively, the truncation map $\pi^{m}: \mathcal{J}_{m}(X) \longrightarrow X$ is induced by the inclusion

$$
k\left[x_{1}, \ldots, x_{n}\right] \hookrightarrow k\left[x_{1}^{(0)}, \ldots, x_{n}^{(0)}, \ldots \ldots, x_{1}^{(m)}, \ldots, x_{n}^{(m)}\right]
$$

sending $x_{i}$ to $x_{i}^{(0)}$.

Exercise 2.5. Let $Y \subseteq \mathbb{A}^{n}$ be a hypersurface given by the vanishing of one equation $f=0$. Show that $\mathcal{J}_{m}(Y) \subseteq \mathcal{J}_{m}\left(\mathbb{A}^{n}\right)$ is given by the vanishing of $m+1$ equations $f^{(0)}, \ldots, f^{(m)}$ in the coordinates of $\mathcal{J}_{m}\left(\mathbb{A}^{n}\right)$ described above. (Observe that $f^{(0)}=f\left(x^{(0)}\right)$ and $\left.f^{(1)}=\sum \frac{\partial}{\partial x_{i}} f\left(x^{(0)}\right) x_{i}^{(1)}\right)$. Show that

(1) $\mathcal{J}_{m}(Y)$ is pure dimensional if and only if $\operatorname{dim} \mathcal{J}_{m}(Y)=(m+1)(n-1)$, in which case $\mathcal{J}_{m}(Y)$ is a complete intersection.

(2) $\mathcal{J}_{m}(Y)$ is irreducible if and only if $\operatorname{dim}\left(\pi_{Y}^{m}\right)^{-1}\left(Y_{\text {Sing }}\right)<(m+1)(n-1)$.

Similar statements hold if $Y$ is locally a complete intersection.

The existence of the jet schemes in general (that is to show the representability of the functor defined above) is proved, for example, in [5]. From the very definition one can easily derive the following étale invariance of jet schemes, which, together with

\footnotetext{
${ }^{15}$ For this to be defined one crucially uses that the restriction maps are affine morphisms.

${ }^{16}$ For this observe that $\lim _{\longleftarrow} \operatorname{Hom}\left(R, k[t] / t^{m+1}\right) \cong \operatorname{Hom}\left(R, \underset{\varliminf}{\lfloor} k[t] / t^{m+1}\right)=\operatorname{Hom}(R, k \llbracket t \rrbracket)$.
} 
the example of $\mathbb{A}^{n}$ above gives us a pretty good understanding of the jet schemes of a smooth variety.

Proposition 2.2. Let $X \rightarrow Y$ be étale, then $\mathcal{J}_{m}(X) \cong \mathcal{J}_{m}(Y) \times_{Y} X$.

Proof. We show the equality on the level of the corresponding functors of points

$$
\operatorname{Hom}\left(\ldots, \mathcal{J}_{m}(X)\right) \cong \operatorname{Hom}\left(\ldots \times_{k} \operatorname{Spec} \frac{k \llbracket t \rrbracket}{t^{m+1}}, X\right)
$$

and

$$
\begin{aligned}
\operatorname{Hom}\left(\ldots, \mathcal{J}_{m}(Y) \times_{Y} X\right) & =\operatorname{Hom}\left(\ldots, \mathcal{J}_{m}(Y)\right) \times \operatorname{Hom}(\ldots, X) \\
& =\operatorname{Hom}\left(\_\times_{k} \operatorname{Spec} \frac{k \llbracket t \rrbracket}{t^{m+1}}, Y\right) \times \operatorname{Hom}(\ldots, X) .
\end{aligned}
$$

For this let $Z$ be a $k$-scheme and consider the diagram

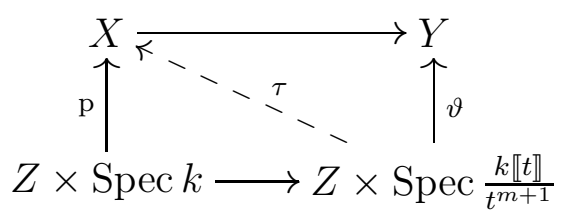

to see that a $Z$-valued $m$-jet $\tau \in \operatorname{Hom}\left(Z \times_{k} \operatorname{Spec} \frac{k \llbracket t \rrbracket}{t^{m+1}}, X\right)$ of $X$ induces a $Z$-valued $m$-jet $\vartheta \in \operatorname{Hom}\left(Z \times_{k} \operatorname{Spec} \frac{k \llbracket t \rrbracket}{t^{m+1}}, Y\right)$ and a map $p \in \operatorname{Hom}(Z, X)$. Virtually by definition formally étaleness [23, Definition (17.1.1)] for the map from $X$ to $Y$, the converse holds also, i.e. $\vartheta$ and $p$ together induce a unique map $\tau$ as indicated.

Using this étale invariance of jet schemes the computation carried out for $\mathbb{A}^{n}$ above holds locally on any smooth $X$. Thus we obtain:

Proposition 2.3. Let $X$ be a smooth $k$-scheme of dimension $n$. Then $\mathcal{J}_{m}(X)$ is locally an $\mathbb{A}^{n m}$-bundle over $X$. In particular $\mathcal{J}_{m}(X)$ is smooth of dimension $n(m+1)$. In the same way, $\mathcal{J}_{m+1}(X)$ is locally an $\mathbb{A}^{n}$-bundle over $\mathcal{J}_{m}(X)$.

Note that this is not true for a singular $X$ as can be seen already by looking at the tangent bundle $T X=\mathcal{J}_{1}(X)$ which is well-known to be a bundle if and only if $X$ is smooth. In fact, over a singular $X$ the jet schemes need not even be irreducible nor reduced and can also be badly singular.

2.3. An algebra of measurable sets. The prototype of a measurable subset of $\mathcal{J}_{\infty}(X)$ is a stable set. They are defined just right so that they receive a natural volume in $\mathcal{M}_{k}$.

Definition 2.4. A subset $A \subseteq \mathcal{J}_{\infty}(X)$ is called stable if for all $m \gg 0, A_{m} \stackrel{\text { def }}{=} \pi_{m}(A)$ is a constructible subset ${ }^{17}$ of $\mathcal{J}_{m}(X), A=\pi_{m}^{-1}\left(A_{m}\right)$ and the map

$$
\pi_{m}^{m+1}: A_{m+1} \rightarrow A_{m} \text { is a locally trivial } \mathbb{A}^{n} \text {-bundle. }
$$

\footnotetext{
${ }^{17}$ The constructible subsets of a scheme $Y$ are the smallest algebra of sets containing the closed sets in Zariski topology.
} 
For any $m \gg 0$ we define the volume of the stable set $A$ by

$$
\mu_{X}(A)=\left[A_{m}\right] \cdot \mathbb{L}^{-n m} \in \mathcal{M}_{k} .
$$

That this is independent of $m$ is ensured by condition (2) which implies that $\left[A_{m+1}\right]=$ $\left[A_{m}\right] \cdot \mathbb{L}^{n} \cdot{ }^{18}$

Assuming that $X$ is smooth one uses Proposition 2.3 to show that the collection of stable sets forms an algebra of sets, which means that $\mathcal{J}_{\infty}(X)$ is stable and with $A$ and $A^{\prime}$ stable the sets $\mathcal{J}_{\infty}(X)-A$ and $A \cap A^{\prime}$ are also stable. The smoothness of $X$ furthermore warrants that so called cylinder sets are stable (a cylinder is a set $A=\pi_{m}^{-1} B$ for some constructible $\left.B \subseteq \mathcal{J}_{m}(X)\right)$. Thus in the smooth case condition (2) is superfluous whereas in general it is absolutely crucial. In fact, a main technical point in defining the motivic measure on singular varieties is to show that the class of stable sets can be enlarged to an algebra of measurable sets which contains the cylinders. In particular $\mathcal{J}_{\infty}(X)$ is then measurable. This is achieved as one would expect by declaring a set measurable if it is approximated in a suitable sense by stable sets. This is essentially carried out in [33], though there are some inaccuracies; but everything should be fine if one works over $\mathbb{C}$ and makes some adjustments following $\left[2\right.$, Appendix]. ${ }^{19}$ To avoid these technicalities we assume until the end of this section that $X$ is smooth over the complex numbers $\mathbb{C}$.

2.4. The measurable function associated to a subscheme. From an algebra of measurable sets there arises naturally a notion of measurable function. Since we did not carefully define the measurable sets — we merely described the prototypes — we will for now only discuss an important class of measurable functions.

Let $Y \subseteq X$ be a subscheme of $X$ defined by the sheaf of ideals $I_{Y}$. To $Y$ one associates the function

$$
\operatorname{ord}_{Y}: \mathcal{J}_{\infty}(X) \rightarrow \mathbb{N} \cup\{\infty\}
$$

sending an arc $\vartheta: \mathcal{O}_{X} \longrightarrow k \llbracket t \rrbracket$ to the order of vanishing of $\vartheta$ along $Y$, i.e. to the supremum of all $e$ such that ideal $\vartheta\left(I_{Y}\right)$ of $k \llbracket t \rrbracket$ is contained in the ideal $\left(t^{e}\right)$. Equivalently, $\operatorname{ord}_{Y}(\vartheta)$ is the supremum of all $e$ such that the map

$$
\mathcal{O}_{X} \stackrel{\vartheta}{\longrightarrow} k \llbracket t \rrbracket \rightarrow k \llbracket t \rrbracket / t^{e}
$$

sends $I_{Y}$ to zero. Note that this map is nothing but the truncation $\pi_{e-1}(\vartheta) \in \mathcal{J}_{e-1}(X)$ of $\vartheta .{ }^{20}$ For a $(e-1)$-jet $\gamma \in \mathcal{J}_{e-1}(X)$ to send $I_{Y}$ to zero means precisely that $\gamma \in \mathcal{J}_{e-1}(Y)$.

\footnotetext{
${ }^{18}$ Reid [40], Batyrev [2] and Looijenga [33] use this definition which gives the volume $\mu_{X}\left(\mathcal{J}_{m}(X)\right) \in$ $\mathcal{M}_{k}$ of $X$ virtual dimension $n$. Denef, Loeser [13] and Craw [7] use an additional factor $\mathbb{L}^{-n}$ to give $\mu_{X}\left(\mathcal{J}_{m}(X)\right)$ virtual dimension 0 . It seems to be essentially a matter of taste which definition one uses. Just keep this in mind while browsing through different sources in the literature to avoid unnecessary confusion.

${ }^{19}$ Of course Denef and Loeser also set up motivic integration over singular spaces [12] but their approach differs from the one discussed here in the sense that they assign a volume to the formula defining a constructible set rather than to the set of ( $k$-rational) points itself. Thus they elegantly avoid any problems which arise if $k$ is small.

${ }^{20}$ At this point we better set $\mathcal{J}_{-1}(X)=X$ and $\pi_{-1}=\pi_{0}=\pi$ to avoid dealing with the case $e=0$ separately.
} 
Thus we can rephrase this by saying that $\operatorname{ord}_{Y}(\vartheta)$ is the supremum of all $e$ such that the truncation $\pi_{e-1}(\vartheta)$ lies in $\mathcal{J}_{e-1}(Y)$. Now it is clear that

$$
\begin{aligned}
\operatorname{ord}_{Y}(\vartheta) \neq 0 & \Leftrightarrow \pi(\vartheta) \in Y, \\
\operatorname{ord}_{Y}(\vartheta) \geq s & \Leftrightarrow \pi_{s-1}(\vartheta) \in \mathcal{J}_{s-1}(Y) \text { and } \\
\operatorname{ord}_{Y}(\vartheta)=\infty & \Leftrightarrow \vartheta \in \mathcal{J}_{\infty}(Y) .
\end{aligned}
$$

The functions ord $_{Y}$ just introduced are examples of measurable functions which come up in the applications of motivic integration. For a function to be measurable, one requires that the level sets $\operatorname{ord}_{Y}^{-1}(s)$ are measurable sets, i.e. stable sets (or at least suitably approximated by stable sets). For this consider the $\operatorname{set}^{\operatorname{ord}_{Y}^{-1}}(\geq s)=\{\vartheta \in$ $\left.\mathcal{J}_{\infty}(X) \mid \operatorname{ord}_{Y}(\vartheta) \geq s\right\}$ consisting of all arcs in $X$ which vanish of order at least $s$ along $Y$. By what we just observed $\operatorname{ord}_{Y}^{-1}(\geq s)=\pi_{s-1}^{-1}\left(\mathcal{J}_{s-1}(Y)\right)$ is a cylinder. Therefore, the level set $\operatorname{ord}_{Y}^{-1}(s)$ is also a cylinder equal to

$$
\operatorname{ord}_{Y}^{-1}(\geq s)-\operatorname{ord}_{Y}^{-1}(\geq s+1)=\pi_{s-1}^{-1} \mathcal{J}_{s-1}(Y)-\pi_{s}^{-1} \mathcal{J}_{s}(Y) .
$$

The exception is $s=0$ in which case $\operatorname{ord}_{Y}^{-1}(\geq 0)=\pi^{-1}(X)=\mathcal{J}_{\infty}(X)$ and $\operatorname{ord}_{Y}^{-1}(0)=$ $\pi^{-1}(X)-\pi^{-1}(Y)$.

Note that the level set at infinity,

$$
\operatorname{ord}_{Y}^{-1}(\infty)=\bigcap \operatorname{ord}_{Y}^{-1}(\geq s)=\mathcal{J}_{\infty}(Y)
$$

on the other hand is not a cylinder. ${ }^{21}$ Still, since it is the decreasing intersection of the cylinders $\operatorname{ord}_{Y}^{-1}(\geq s+1)=\pi_{s}^{-1} \mathcal{J}_{s}(Y)$ its alleged volume should be obtained as the limit of the volumes of these cylinders. The volume of $\pi_{s}^{-1} \mathcal{J}_{s}(Y)$ is $\left[\mathcal{J}_{s}(Y)\right] \cdot \mathbb{L}^{-n s}$. The dimension of this element of $\mathcal{M}_{k}$ is $\leq \operatorname{dim} \mathcal{J}_{s}(Y)-n s$. If $Y$ is smooth of dimension $n-c$, the we have seen that $\operatorname{dim} \mathcal{J}_{s}(Y)=(n-c)(s+1)$. Therefore, $\mathcal{J}_{\infty}(Y)$ is the intersection of cylinder sets whose volumes have dimension $\leq(n-c)(s+1)-n s=(n-c)-c s$. For increasing $s$ these dimensions tend to negative infinity. But recall that in the ring $\hat{\mathcal{M}}_{k}$ this is exactly the condition of convergence to zero. Thus the only sensible assignment of a volume to $\operatorname{ord}_{Y}^{-1}(\infty)$ is zero. This argument used that $Y$ is smooth to show that the dimension of $\mathcal{J}_{m}(Y)$ grows significantly slower than the dimension of $\mathcal{J}_{m}(X)$. This holds in general for singular $Y$ and we phrase it as a proposition whose proof however is postponed until Section 4.

Proposition 2.5. Let $Y \subseteq X$ be a nowhere dense subvariety of $X$, then $\mathcal{J}_{\infty}(Y)$ is measurable and has measure $\mu_{X}\left(\mathcal{J}_{\infty}(Y)\right)$ equal to zero.

To make this idea into a rigorous theory one has to define a larger class of measurable subsets of $\mathcal{J}_{\infty}(X)$ and this turns out to be somewhat subtle. In section 4 we will outline this briefly - but since, no matter what, the measure of $\operatorname{ord}_{Y}^{-1}(\infty)$ will be zero, we will move on at this point and start to integrate.

\footnotetext{
${ }^{21}$ This just says that for an $\operatorname{arc} \vartheta \in \mathcal{J}_{\infty}(X)$ to lie in $\mathcal{J}_{\infty}(Y)$ is a condition that cannot be checked on any truncation. Exercise!
} 
2.5. Definition and computation of the motivic integral. As before let $X$ be a smooth $k$-scheme and $Y$ a subscheme. We define the motivic integral of $\mathbb{L}^{-\operatorname{ord}_{Y}}$ on $X$ as

$$
\int_{\mathcal{J}_{\infty}(X)} \mathbb{L}^{-\operatorname{ord}_{Y}} d \mu_{X}=\sum_{s=0}^{\infty} \mu\left(\operatorname{ord}_{Y}^{-1}(s)\right) \cdot \mathbb{L}^{-s} .
$$

Observe that the level set at infinity is already left out from this summation as it has measure zero.

Note that the sum on the right does converge since the virtual dimension of the summands approaches negative infinity. ${ }^{22}$ The notion of convergence in the ring $\hat{\mathcal{M}}_{k}$ is such that this alone is enough to ensure the convergence of the sum. Thus it is justified to call $\mathbb{L}^{- \text {ord }_{Y}}$ integrable with integral as above.

It is useful to calculate at least one example. For $Y=\emptyset$ one has $\operatorname{ord}_{Y} \equiv 0$ and thus we get

$$
\int_{\mathcal{J}_{\infty}(X)} \mathbb{L}^{-\operatorname{ord}_{Y}} d \mu_{X}=\mu\left(\operatorname{ord}_{Y}^{-1}(0)\right)=[X]
$$

where we used that $X$ is smooth. A less trivial example is $Y$ a smooth divisor in $X$. Then $\mathcal{J}_{s}(Y)$ is locally a $\mathbb{A}^{(n-1) s}$-bundle over $Y$. The level set is $\operatorname{ord}_{Y}^{-1}(s)=$ $\pi_{s-1}^{-1} \mathcal{J}_{s-1}(Y)-\pi_{s}^{-1} \mathcal{J}_{s}(Y)$ and, using that $\left[\mathcal{J}_{s}(Y)\right]=[Y] \cdot \mathbb{L}^{(n-1) s}$, its measure is

$$
\left[\mathcal{J}_{s-1}(Y)\right] \cdot \mathbb{L}^{-n(s-1)}-\left[\mathcal{J}_{s}(Y)\right] \cdot \mathbb{L}^{-n s}=[Y](\mathbb{L}-1) \mathbb{L}^{-s} .
$$

The integral of $\operatorname{ord}_{Y}^{-1}$ is therefore

$$
\begin{aligned}
\int_{\mathcal{J}_{\infty}(X)} \mathbb{L}^{-\operatorname{ord}_{Y}} d \mu_{X} & =[X-Y]+\sum_{s=1}^{\infty}[Y](\mathbb{L}-1) \mathbb{L}^{-s} \cdot \mathbb{L}^{-s} \\
& =[X-Y]+[Y](\mathbb{L}-1) \mathbb{L}^{-2} \sum_{s=0}^{\infty} \mathbb{L}^{-2 s} \\
& =[X-Y]+[Y](\mathbb{L}-1) \frac{1}{\mathbb{L}^{2}\left(1-\mathbb{L}^{-2}\right)} \\
& =[X-Y]+[Y](\mathbb{L}-1)\left(\mathbb{L}^{2}-1\right)^{-1} \\
& =[X-Y]+\frac{[Y]}{\mathbb{L}+1}=[X-Y]+\frac{[Y]}{\left[\mathbb{P}^{1}\right]}
\end{aligned}
$$

Note the appearance of a geometric series in line 3 which is typical for these calculations (cf. Exercise 2.4). In fact, the motivic volumes of a wide class of measurable subsets (namely, the semi-algebraic subsets of Denef and Loeser in [12]) belong to the ring generated by the image of $\mathcal{M}_{k}$ under the completion map, and the sums of geometric series with denominators $\mathbb{L}^{j}, j>0[11$, Corollary 5.2].

\footnotetext{
${ }^{22}$ The fact that for stable sets $A \subseteq B$ we have $\operatorname{dim} \mu(A) \leq \operatorname{dim} \mu(B)$ applied to $\operatorname{ord}_{Y}^{-1}(s) \subseteq \mathcal{J}_{\infty}(X)$ gives that the dimension of $\mu\left(\operatorname{ord}_{Y}^{-1}(s)\right) \cdot \mathbb{L}^{-s}$ is less or equal to $n-s$.
} 
Exercise 2.6. ${ }^{23}$ Compute in a similar fashion the motivic integrals $\mathbb{L}^{-\operatorname{ord}_{Y}}$ where $Y$ is as follows.

a) $Y$ is a smooth subscheme of codimension $c$ in $X$.

b) $Y=a D$ where $D$ is a smooth divisor and $a \in \mathbb{N}$.

c) $Y=D_{1}+D_{2}$ where the $D_{i}$ 's are smooth and in normal crossing.

d) $Y=a_{1} D_{1}+a_{2} D_{2}$ with $D_{i}$ as above and $a_{i}$ positive integers.

These computations are a special case of a formula which explicitly computes the motivic integral over $\mathbb{L}^{- \text {ord } Y}$ where $Y$ is an effective divisor with normal crossing support.

Proposition 2.6. Let $Y=\sum_{i=1}^{s} r_{i} D_{i}\left(r_{i}>0\right)$ be an effective divisor on $X$ with normal crossing support and such that all $D_{i}$ are smooth. Then

$$
\int_{\mathcal{J}_{\infty}(X)} \mathbb{L}^{-\operatorname{ord}_{Y}} d \mu_{X}=\sum_{J \subseteq\{1, \ldots, s\}}\left[D_{J}^{\circ}\right]\left(\prod_{j \in J} \frac{\mathbb{L}-1}{\mathbb{L}^{r_{j}+1}-1}\right)=\sum_{J \subseteq\{1, \ldots, s\}} \frac{\left[D_{J}^{\circ}\right]}{\prod_{j \in J}\left[\mathbb{P}^{r_{j}}\right]}
$$

$D_{J}=\bigcap_{j \in J} D_{j}$ (note that $D_{\emptyset}=X$ ) and $D_{J}^{\circ}=D_{J}-\bigcup_{j \notin J} D_{j}$.

The proof of this is a computation entirely similar to (though significantly more complicated than) the one carried out in (3) above; for complete detail see either Batyrev [2, Theorem 6.28] or Craw [7, Theorem 1.17]. We suggest doing it as an exercise using the following lemma.

Lemma 2.7. For $J \subseteq\{1, \ldots, s\}$ redefine $r_{i}=0$ if $i \notin J$. Then

$$
\mu\left(\cap \operatorname{ord}_{D_{j}}^{-1}\left(r_{j}\right)\right)=\left[D_{J}^{0}\right](\mathbb{L}-1)^{|J|} \mathbb{L}^{-\sum r_{j}}
$$

where $D_{J}^{0}=\cap_{j \in J} D_{j}-\cup_{j \notin J} D_{J}$ and $|J|$ denotes the cardinality of $J$.

Exercise 2.7. ${ }^{24}$ Show that for $t \geq \max \left\{r_{i}\right\}$ one has locally an isomorphism of the $k$-points

$$
\pi_{t}\left(\cap_{i} \operatorname{ord}_{D_{i}}^{-1}\left(r_{i}\right)\right) \cong D_{\operatorname{supp} r}^{0} \times(k-\{0\})^{|\operatorname{supp} r|} \times k^{n t-\sum r_{i}} .
$$

${ }^{23}$ The answer in the first case is $[X-Y]+[Y] \frac{\mathbb{L}^{c}-1}{\mathbb{L}^{c+1}-1}$. Otherwise it can be read off from the general formula below; e.g. in the second case it is $[X-D]+\frac{[D]}{\left[\mathbb{P}^{a}\right]}$.

${ }^{24}$ Hint/Solution: Clearly, for an arc $\vartheta$ membership in $\cap \operatorname{ord}_{D_{i}}^{-1}\left(r_{i}\right)$ only depends on its truncation $\pi_{t}(\vartheta)$ as long as $t \geq \max \left\{r_{i}\right\}$. With the notation of Example 2.1 we write

$$
\pi_{t}\left(\vartheta\left(x_{i}\right)\right)=\sum_{j=0}^{t} \vartheta_{i}^{(j)} t^{j}
$$

such that $\vartheta$ is determined by the coefficients $\vartheta_{i}^{(j)}$. Now we determine what the condition $\vartheta \in$ $\cap \operatorname{ord}_{D_{i}}^{-1}\left(r_{i}\right)$ imposes on the coefficients $\vartheta_{i}^{(j)}$ (here it is convenient to set $r_{i}=0$ for $i>s$ ).

(1) For $j<r_{i}$ one has $\vartheta_{i}^{(j)}=0$.

(2) For $j=r_{i}$ one has $\vartheta_{i}^{(j)} \neq 0$.

(3) For $j>r_{i}$ one has no condition $\vartheta_{i}^{(j)}$.

Thus the set $\pi_{t}\left(\cap_{i} \operatorname{ord}_{D_{i}}^{-1}\left(r_{i}\right)\right)$ is the product made up form the factors $D_{\operatorname{supp} r}^{0}$ corresponding to all possible $\vartheta_{i}^{(0)}$, a copy of $k-\{0\}$ for each possible $\vartheta_{i}^{\left(r_{i}\right)}$ and $r_{i}>0$ and a copy of $k$ for each $r_{i}<j \leq k$. Putting this together we obtain the above formula. 
Show that this implies the lemma. To prove the preceding statement reduce to the case that $X=U \subseteq \mathbb{A}^{n}$ is an open subvariety of $\mathbb{A}^{n}$ and $\sum D_{i}$ is given by the vanishing of $x_{1} \cdot \ldots \cdot x_{s}$ where $x_{1}, \ldots, x_{n}$ is a local system of coordinates (use Proposition 2.2). Then finish this case using the description of the arc space of $\mathbb{A}^{n}$ as given in Example 2.1.

The explicit formulas of Proposition 2.6 and Lemma 2.7 are one cornerstone underlying many applications of motivic integration. The philosophy one employs is to encode information in a motivic integral, then using the transformation rule of the next section the computation of this integral can be reduced to the computation of an integral over $\mathbb{L}^{- \text {ord }_{Y}}$ for $Y$ a normal crossing divisor. In this case the above formula gives the answer. Thus we shall proceed to the all important birational transformation rule for motivic integrals.

\section{The transformation RULE}

The power of the theory stems from a formula describing how the motivic integral transforms under birational morphisms:

Theorem 3.1. Let $X^{\prime} \stackrel{f}{\longrightarrow} X$ be a proper birational morphism of smooth $k$-schemes and let $D$ be an effective divisor on $X$, then

$$
\int_{\mathcal{J}_{\infty}(X)} \mathbb{L}^{-\operatorname{ord}_{D}} d \mu_{X}=\int_{\mathcal{J}_{\infty}\left(X^{\prime}\right)} \mathbb{L}^{-\operatorname{ord}_{f^{-1} D+K_{X^{\prime}} / X}} d \mu_{X^{\prime}}
$$

As the relative canonical sheaf $K_{X^{\prime} / X}$ is defined by the Jacobian ideal of $f$, this should be thought of as the change of variables formula for the motivic integral.

As a warmup for the proof, we verify the the transformation rule first in the special case of blowing up a smooth subvariety and $D=\emptyset$. Let $X^{\prime}=\mathrm{Bl}_{Y} X$ be the blowup of $X$ along the smooth center $Y$ of codimension $c$ in $X$. Then by [26, Exercise II.8.5] the relative canonical divisor is $K_{X^{\prime} / X}=(c-1) E$, where $E$ is the exceptional divisor of the blowup. Then, using Proposition 2.6 in its simplest incarnation we compute

$$
\begin{aligned}
\int_{\mathcal{J}_{\infty}\left(X^{\prime}\right)} \mathbb{L}^{-\operatorname{ord}_{K_{X^{\prime}} / X} d \mu_{X^{\prime}}} & =\int_{\mathcal{J}_{\infty}\left(X^{\prime}\right)} \mathbb{L}^{-\operatorname{ord}_{(c-1) E}} d \mu_{X^{\prime}} \\
& =\left[X^{\prime}-E\right]+\frac{[E]}{[\mathbb{P} c]} \\
& =[X-Y]+[Y]=[X],
\end{aligned}
$$

where we used that $E$ is a $\mathbb{P}^{c}$-bundle over $Y$ (by definition of blowup) and therefore $[E]=[Y]\left[\mathbb{P}^{c}\right]$.

3.0.1. The induced map on the arc space. The proper birational map $f$ induces a map $f_{\infty}=\mathcal{J}_{\infty}(f): \mathcal{J}_{\infty}\left(X^{\prime}\right) \longrightarrow \mathcal{J}_{\infty}(X)$. The first task will be to show that away from a set of measure zero $f_{\infty}$ is a bijection (of sets). Let $\Delta \subseteq X^{\prime}$ be the locus where $f$ is not an isomorphism. We show that every arc $\gamma: \operatorname{Spec} k \llbracket t \rrbracket \rightarrow X$, which does not entirely lie 
in $f(\Delta)$ uniquely lifts to an arc in $X^{\prime}$. For illustration consider the diagram

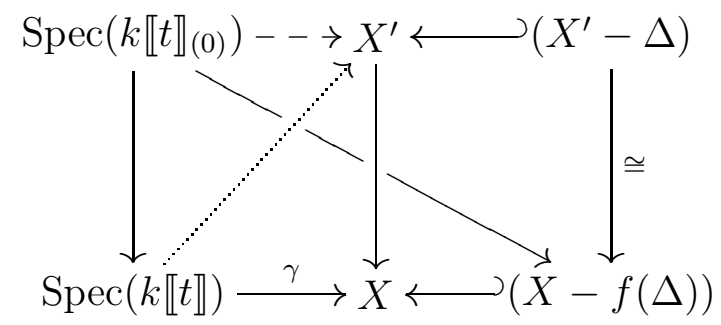

Observe that by assumption the generic point Spec $k \llbracket t \rrbracket_{(0)}$ of Spec $k \llbracket t \rrbracket$ does lie in $X-f(\Delta)$ and since $f$ is an isomorphism over $X^{\prime}-\Delta$ it thus lifts to $X^{\prime}$ uniquely (dashed arrow). Now the valuative criterion for properness (see [26, Chapter 2, Theorem 4.7]) yields the unique existence of the dotted arrow. Thus the map $f_{\infty}$ : $\left(\mathcal{J}_{\infty}\left(X^{\prime}\right)-\mathcal{J}_{\infty}(\Delta)\right) \rightarrow\left(\mathcal{J}_{\infty}(X)-\mathcal{J}_{\infty}(f(\Delta))\right)$ is a bijection of $k$-valued points. Since $\mathcal{J}_{\infty}(\Delta)$ has measure zero it can be safely ignored and we will do so in the following.

Exercise 3.1. ${ }^{25}$ Let $f: X^{\prime} \longrightarrow X$ be a proper birational map (of smooth varieties). Then for every $m$ the map $f: \mathcal{J}_{m}\left(X^{\prime}\right) \longrightarrow \mathcal{J}_{m}(X)$ is surjective.

Proof of transformation rule. The level sets $C_{e}^{\prime}=\operatorname{ord}_{K_{X^{\prime} / X}}^{-1}(e)$ partition $\mathcal{J}_{\infty}\left(X^{\prime}\right)$, and cutting into even smaller pieces according to order of contact along $f^{-1}(D)$ we define

$$
C_{e, k}^{\prime}=C_{e}^{\prime} \cap \operatorname{ord}_{f^{-1} D}^{-1}(k) \text { and } C_{e, k}=f_{\infty}\left(C_{e, k}^{\prime}\right)
$$

to get the following partitions (up to measure zero by 3.0.1) of the arc spaces

$$
\mathcal{J}_{\infty}\left(X^{\prime}\right)=\bigsqcup C_{e, k}^{\prime} \text { and } \mathcal{J}_{\infty}(X)=\bigsqcup C_{e, k} .
$$

The essence of the proof of the transformation rule is captured by the following two crucial facts.

(a) $C_{e, k}$ are stable sets for all $e, k$.

(b) $\mu_{X}\left(C_{e, k}\right)=\mu_{X^{\prime}}\left(C_{e, k}^{\prime}\right) \cdot \mathbb{L}^{-e}$.

Two different proofs of these facts will occupy the remainder of this section. Using these facts, the transformation rule is a simple calculation:

$$
\begin{aligned}
\int_{\mathcal{J}_{\infty}(X)} \mathbb{L}^{-\operatorname{ord}_{D} d \mu_{X}} & =\sum_{k} \mu\left(\operatorname{ord}_{D}^{-1}(k)\right) \mathbb{L}^{-k}=\sum_{k}\left(\sum_{e} \mu\left(C_{e, k}\right)\right) \mathbb{L}^{-k} \\
& =\sum_{e, k} \mu\left(C_{e, k}^{\prime}\right) \mathbb{L}^{-e} \mathbb{L}^{-k} \\
& =\sum_{t}\left(\sum_{e+k=t} \mu\left(C_{e, k}^{\prime}\right)\right) \mathbb{L}^{-t}=\sum_{t} \mu\left(\operatorname{ord}_{f^{-1} D+K_{X^{\prime} / X}}^{-1}(t)\right) \mathbb{L}^{-t} \\
& =\int_{\mathcal{J}_{\infty}\left(X^{\prime}\right)} \mathbb{L}^{-\operatorname{ord}_{f^{-1} D+K_{X^{\prime} / X}} d \mu_{X^{\prime}}}
\end{aligned}
$$

\footnotetext{
${ }^{25}$ Solution: The valuative criterion of properness shows that any $\gamma \notin \mathcal{J}_{\infty}(Z)$ lies in the image (where $Z \subseteq X$ is such that $f$ is an isomorphism from $X^{\prime}-f^{-1}(Z) \rightarrow X-Z$. But for any $\gamma_{m} \in \mathcal{J}_{m}(X)$ the cylinder $\pi_{m}^{-1}\left(\gamma_{m}\right)$ cannot be contained in $\mathcal{J}_{\infty}(Z)$ since the latter has measure zero.
} 
Besides facts (a) and (b) one uses that if a cylinder $B$ is written as a disjoint union of cylinders $B_{i}$ then the measure $\mu(B)=\sum \mu\left(B_{i}\right)$. To check that this is correct one has to use the precise definition of the motivic measure which we have avoided until now. See the Section 4 for more details on this.

We first treat properties (a) and (b) in a special case, namely the blowup at a smooth center. In the applications of motivic integration to birational geometry which we discuss below, we can always put ourselves in the favorable situation that the birational map in consideration is a sequence of blowing ups along smooth centers, hence already this simple version goes very far. Furthermore, using the Weak Factorization Theorem of [1] one can make a general proof of the transformation rule by reducing to this case. However, despite the adjective weak in the Weak Factorization Theorem it is a very deep and difficult result and its use in the proof of the Transformation rule is overkill. Therefore we give in Appendix A.2 an essentially elementary (though at the first reading somewhat technical) proof following [33].

3.1. Images of cylinders under birational maps. We start with some basic properties of the behaviour of cylinders under birational morphisms, see [14]. These will be useful also in Section 5.

Proposition 3.2. Let $f: X^{\prime} \longrightarrow X$ be a proper birational map of smooth varieties. Let $C^{\prime}=\left(\pi_{m}^{X^{\prime}}\right)^{-1}\left(B^{\prime}\right) \subseteq \mathcal{J}_{\infty}\left(X^{\prime}\right)$ be a cylinder such that $B^{\prime}$ is a union of fibers of $f_{m}$, then $C \stackrel{\text { def }}{=} f_{\infty}\left(C^{\prime}\right)=\left(\pi_{m}^{X}\right)^{-1}\left(f_{m} B^{\prime}\right)$ is a cylinder in $\mathcal{J}_{\infty}(X)$.

Proof. Clearly it is enough to show that $C=\pi_{m}^{-1}(B)$ since $B=f_{m}\left(B^{\prime}\right)$ is constructible (being the image of a constructible set under a finite type morphism). The nontrivial inclusion is $\pi_{m}^{-1}(B) \subseteq C$. Let $\gamma \in \pi_{m}^{-1}(B) \subseteq C$ and consider for every $p \geq m$, the cylinder

$$
D_{p} \stackrel{\text { def }}{=}\left(\pi_{p}^{X^{\prime}}\right)^{-1}\left(f_{p}^{-1}\left(\pi_{p}^{X}(\gamma)\right)\right)
$$

which is non empty by exercise 3.1. Clearly, $D_{p} \supseteq D_{p+1}$ which implies that the $D_{p}$ form a decreasing sequence of nonempty cylinders. By Proposition 4.3 the intersection of all the $D_{p}$ is nonempty. Now let $\gamma^{\prime} \in \bigcap D_{p}$ and clearly $f_{\infty} \gamma^{\prime}=\gamma$. Furthermore, since $B^{\prime}$ is a union of fibers of $f_{m}$ we have $C^{\prime} \supseteq D_{m}$ and hence $C^{\prime} \supseteq D_{p}$ for all $p \geq m$. Hence $\pi_{m}\left(\gamma^{\prime}\right) \in B^{\prime}$.

Exercise 3.2. ${ }^{26}$ Let $f: X^{\prime} \longrightarrow X$ be a proper birational map of smooth varieties. Then $f_{\infty}: \mathcal{J}_{\infty}\left(X^{\prime}\right) \longrightarrow \mathcal{J}_{\infty}(X)$ is surjective.

3.1.1. The key technical result for blowup at smooth center. We now proceed to showing the key technical result used in the proof of the transformation formula.

Theorem 3.3 (Denef, Loeser, [12]). Let $f: X^{\prime} \longrightarrow X$ be a proper birational morphism of smooth varieties. Let $C_{e}^{\prime}=\operatorname{ord}_{K_{X^{\prime} / X}}^{-1}(e)$ where $K_{X^{\prime} / X}$ is the relative canonical divisor and let $C_{e} \stackrel{\text { def }}{=} f_{\infty} C_{e}^{\prime}$. Then, for $m \geq 2 e$

\footnotetext{
${ }^{26}$ Solution: The previous proposition shows that $f_{\infty}\left(\mathcal{J}_{\infty}\left(X^{\prime}\right)\right)$ is a cylinder. The surjectivity of $f_{m}$ which was showed in exercise 3.1 now implies the result (in fact even the surjectivity of $f_{0}=f$ is enough at this point).
} 
(a') the fiber of $f_{m}$ over a point $\gamma_{m} \in \pi_{m} C_{e}$ lies inside a fiber of $\pi_{m-e}^{m}$.

(a) $\pi_{m}\left(C_{e}^{\prime}\right)$ is a union of fibers of $f_{m}$.

(b) $f_{m}: \pi_{m} C_{e}^{\prime} \longrightarrow \pi_{m} C_{e}$ is piecewise trivial $\mathbb{A}^{e}$-fibration.

Corollary 3.4. With the notation as in the theorem, $C_{e}$ is a stable at level $m \geq 2 e$ and $\left[C_{e}^{\prime}\right]=\left[C_{e}\right] \mathbb{L}^{e}$.

Proof of Theorem 3.3. First note (a') implies (a) since $C_{e}^{\prime}$ is stable at level $e$. Furthermore using the following Lemma 3.5 it is enough to show that a fibers of $f_{m}$ over a point in $C_{e}$ is affine space $\mathbb{A}^{e}$.

We give the proof here only in the case that $X^{\prime}=\mathrm{Bl}_{Y} X \rightarrow X$ where $Y$ is a smooth subvariety of $X$, for the general argument see Appendix A.2 below. Since $X$ is smooth there is an étale morphism $\varphi: X \rightarrow \mathbb{A}^{n}$ such that $\varphi(Y)$ is given by the vanishing of the first $n-c$ coordinates on $\mathbb{A}^{n}$ and $\varphi^{-1}(\varphi(Y))=Y$. By the étale invariance of jet schemes (Proposition 2.2) one can hence assume that $Y=\mathbb{A}^{n-c} \subseteq \mathbb{A}^{n}=X{ }^{27}$

To further simplify notation (and notation only) we assume that $n=3$ and $c=3$, that is we only have to consider the blowup of the origin in $\mathbb{A}^{3}$. By definition

$$
X^{\prime}=\mathrm{Bl}_{0} \mathbb{A}^{3} \subseteq \mathbb{A}^{3} \times \mathbb{P}^{2}
$$

is given by the vanishing of the $2 \times 2$ minors of the matrix

$$
\left(\begin{array}{lll}
x_{0} & x_{1} & x_{2} \\
y_{0} & y_{1} & y_{2}
\end{array}\right)
$$

where $\left(x_{0}, x_{1}, x_{2}\right)$ and $\left(z_{0}, z_{1}, z_{2}\right)$ are the coordinates on $\mathbb{A}^{3}$ and the homogeneous coordinates of $\mathbb{P}^{2}$ respectively. Due to the local nature of our question it is enough to consider on affine patch of $X^{\prime}$, say the one determined by $z_{0}=1$. The equations of the minors then reduce to $x_{1}=x_{0} z_{1}$ and $x_{2}=x_{0} z_{2}$ such that on this patch the map $X^{\prime} \longrightarrow X$ is given by the inclusion of polynomial rings

$$
k\left[x_{0}, x_{0} z_{1}, x_{0} z_{2}\right] \stackrel{f}{\longrightarrow} k\left[x_{0}, z_{1}, z_{2}\right] .
$$

The exceptional divisor $E$ is hence given by the vanishing of $x_{0}$. The relative canonical divisor $K_{X^{\prime} / X}$ is equal to $2 E$ since $\operatorname{det}(\operatorname{Jac}(f))$ is easily computed to be $x_{0}^{2}$.

${ }^{27}$ This is not as straight froward as as it might seem. One has to check that $\mathrm{Bl}_{Y} X \cong X \times{ }_{\mathbb{A}}^{n} \mathrm{Bl}_{\varphi(Y)} \mathbb{A}^{n}$. Let $I$ be the ideal defining $Y$ and $\varphi_{*}(I)$ the ideal defining $\varphi(Y)$. Locally one has $\varphi^{*}\left(\varphi_{*}(I)\right)=I$. The pullback of $\varphi_{*}(I)$ to $\mathrm{Bl}_{Y} X$ is equal to the pullback of $I$ which is by construction of the blow-up a principal ideal sheaf. By the universal property of the blowup (applied to $\mathrm{Bl}_{\varphi(Y)} \mathbb{A}^{n}$ ) we get a map $\mathrm{Bl}_{Y} X \rightarrow \mathrm{Bl}_{\varphi(Y)} \mathbb{A}^{n}$ and hence a map

$$
\mathrm{Bl}_{Y} X \longrightarrow \mathrm{Bl}_{\varphi(Y)} \mathbb{A}^{n} \times_{\mathbb{A}^{n}} X
$$

The pullback of $I$ to $\mathrm{Bl}_{\varphi(Y)} \mathbb{A}^{n} \times_{\mathbb{A}^{n}} X$ along the second projection is equal to the pullback of $\varphi_{*}(I)$ along the natural map to $\mathbb{A}^{n}$ which factors through the blowup. Hence this pullback is locally principal and again by the universal property of blowup (applied to $\mathrm{Bl}_{Y} X \rightarrow X$ ) we get a map in the opposite direction, which is easily verified to be inverse to the one given above. This implies in particular that $\mathrm{Bl}_{Y} X$ is étale over $\mathrm{Bl}_{\varphi(Y)} \mathbb{A}^{n}$ and thus

$$
\mathcal{J}_{m}\left(\mathrm{Bl}_{Y} X\right) \cong \mathrm{Bl}_{Y} X \times_{\mathrm{Bl}_{\varphi}(Y)} \mathbb{A}^{n} \mathcal{J}_{m}\left(\mathrm{Bl}_{\varphi(Y)} \mathbb{A}^{n}\right) \cong X \times_{\mathbb{A}^{n}} \mathcal{J}_{m}\left(\mathrm{Bl}_{\varphi(Y)} \mathbb{A}^{n}\right) .
$$

This allows one after fixing the base point $x \in X$ of $\gamma$ to really reduce to the case of affine space. 
Now let $\gamma^{\prime} \in C_{e}^{\prime}$. In our local coordinates, $\gamma_{m}^{\prime}=\pi_{m}\left(\gamma^{\prime}\right)$ is uniquely determined by the three truncated powerseries

$$
\begin{aligned}
\gamma_{m}^{\prime}\left(x_{0}\right) & =t^{e / 2} \sum_{i=0}^{m-e / 2} a_{i} t^{i} \quad \text { with } a_{0} \neq 0 \\
\gamma_{m}^{\prime}\left(z_{1}\right) & =\sum_{i=0}^{m} b_{i} t^{i} \text { and } \\
\gamma_{m}^{\prime}\left(z_{2}\right) & =\sum_{i=0}^{m} c_{i} t^{i},
\end{aligned}
$$

where the special shape of the first one comes from the condition that $\gamma^{\prime}$ has contact order with $K_{X^{\prime} / X}=2 E$ precisely equal to $e$ (we only have to consider $e$ which are divisible by $c-1=2$ since otherwise $C_{e}^{\prime}$ is empty). Its image $\gamma_{m}=f_{m}\left(\gamma_{m}^{\prime}\right)=\gamma_{m}^{\prime} \circ f_{m}$ is analogously determined by the three truncated powerseries

$$
\begin{gathered}
\gamma_{m}\left(x_{0}\right)=t^{e / 2} \sum_{i=0}^{m-e / 2} a_{i} t^{i} \quad \text { with } a_{0} \neq 0 \\
\gamma_{m}\left(x_{0} z_{1}\right)=\gamma_{m}^{\prime}\left(x_{0}\right) \gamma_{m}^{\prime}\left(z_{1}\right)=t^{e / 2} \sum_{i=0}^{m-e / 2} a_{i} t^{i} \sum_{i=0}^{m} b_{i} t^{i} \bmod t^{m+1} \\
\gamma_{m}\left(x_{0} z_{2}\right)=\gamma_{m}^{\prime}\left(x_{0}\right) \gamma_{m}^{\prime}\left(z_{1}\right)=t^{e / 2} \sum_{i=0}^{m-e / 2} a_{i} t^{i} \sum_{i=0}^{m} c_{i} t^{i} \bmod t^{m+1}
\end{gathered}
$$

Expanding the product of the sums in the last two equations of (4) we observe that (due to the occurence of $t^{e / 2}$ ) the coefficients $b_{m-\frac{e}{2}+1}, \ldots, b_{m}$ are not visible in $\gamma_{m}\left(x_{0} z_{1}\right)$ since they only appear as coefficients of some $t^{k}$ for $k>m$. Analogously, $\gamma_{m}\left(x_{0} z_{2}\right)$ does not depend on $c_{m-\frac{e}{2}+1}, \ldots, c_{m}$. Conversely, given

$$
\gamma_{m}\left(x_{0} z_{1}\right)=t^{e / 2} \sum_{i=0}^{m-\frac{e}{2}} \beta_{i} t^{i}
$$

and knowing all $a_{i}$ 's (which equally show up in $\gamma_{m}^{\prime}$ and $\gamma_{m}$ ) we can inductively recover the $b_{i}$ 's:

$$
\begin{aligned}
b_{0} & =\left(\beta_{0}\right) / a_{0} \quad\left(\text { note: } a_{0} \neq 0\right) \\
b_{1} & =\left(\beta_{1}-a_{1} b_{0}\right) / a_{0} \\
b_{2} & =\left(\beta_{2}-\left(a_{2} b_{0}+a_{1} b_{1}\right)\right) / a_{0} \\
\vdots & \\
b_{t} & =\left(\beta_{t}-\left(a_{t} b_{0}+a_{t-1} b_{1}+\ldots+a_{1} b_{t-1}\right)\right) / a_{0}
\end{aligned}
$$

and this works until $t=m-\frac{e}{2}$ since $a_{t}$ is known for $t \leq m-\frac{e}{2}$. The analogous statements of course hold also for the $c_{i}$ 's. 
Summing up these observations we see that the fiber of $f_{m}$ over $\gamma_{m}$ is an affine space of dimension $e=2 \cdot \frac{e}{2}$, namely it is spanned by the last $\frac{e}{2}$ of the coefficients $b_{i}$ and $c_{i}$. This proves part (b). Furthermore, any two $\gamma_{m}^{\prime}$ and $\gamma_{m}^{\prime \prime}$ mapping via $f_{m}$ to $\gamma_{m}$ only differ in these last $\frac{e}{2}$ coefficients, hence they become equal after further truncation to level $m-e .{ }^{28}$ This shows $\left(a^{\prime}\right)$ and the proof is finished.

Lemma 3.5. Let $\varphi: V \longrightarrow W$ be a morphism of finite type schemes such that all fibers $\varphi^{-1}(x) \cong \mathbb{A}^{e} \times k(x)$, then $\varphi$ is a piecewise trivial $\mathbb{A}^{e}$-fibration. ${ }^{29}$

Proof. We may assume that $W$ is irreducible. Then the fiber over the generic point $\eta$ of $W$ is by assumption isomorphic to $\mathbb{A}^{e}$. This means that there is an open subset $U \subseteq W$ such that $f^{-1}(U) \cong \mathbb{A}^{e} \times U .{ }^{30}$ Now $\varphi$ restricted to the complement of $U$ is a map of the same type but with smaller dimensional base and we can finish the argument by induction. Even though we did not make this explicit in the proof of Theorem 3.3 the statement about the fibers being equal to $\mathbb{A}^{e}$ holds for all fibers (not only fibers over closed points).

Exercise 3.3. ${ }^{31}$ Use Proposition 3.2 and 3.3 to show that if $C \subseteq \mathcal{J}_{\infty}\left(X^{\prime}\right)$ is a cylinder, then the closure of $f_{\infty}(C) \subseteq \mathcal{J}_{\infty}(X)$ is a cylinder, where $f: X^{\prime} \longrightarrow X$ is a proper birational morphism.

3.2. Proof of transformation rule using Weak Factorization. With the proof given so far we have the transformation formula available for a large class of proper birational morphisms, namely the ones which are obtained as a sequence of blowups along smooth centers. So in particular we have the result for all resolutions of singularities, which is the only birational morphism we will consider in our applications later.

Let me finish by outlining how using the Weak Factorization Theorem one can make a full proof out of this. Let us first recall the statement:

Theorem 3.6 (Weak Factorization Theorem [1]). Let $\varphi: X^{\prime} \rightarrow X$ be a birational map between smooth complete varieties over $k$ of characteristic zero. Then $\varphi$ can be factored

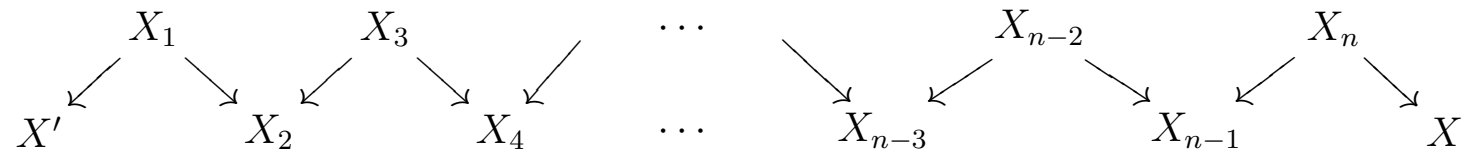

\footnotetext{
${ }^{28}$ Even to level $m-\frac{e}{2}$ in this case of blowup of a point in $\mathbb{A}^{3}$. In general, for a blowup of $c$ codimensional smooth center it is truncation to level $m-\frac{e}{c-1}$ which suffices. Hence uniformly it is truncation to level $m-e$ which works.

${ }^{29}$ In fact, it follows from Hilbert's Theorem 90 that a piecewise trivial $\mathbb{A}^{e}$ fibration is actually locally trivial. EXPLAIN!

${ }^{30}$ The isomorphism $\mathbb{A}^{e}(k(\eta)) \cong \varphi^{-1}(\eta)=V \times{ }_{W} \operatorname{Spec} k(\eta)$ is defined via some finitely many rational functions on $W$. For any $U \subseteq W$ such that these are regular we get $\varphi^{-1}(U) \cong \mathbb{A}^{e} \times U$.

${ }^{31}$ We may assume that $C$ is irreducible. Let $e$ be smallest such that $C \cap$ Cont $_{K_{X^{\prime} / X}}^{e} \neq 0$. This intersection is open and dense in $C$, and hence we may replace $C$ by $C \cap$ Cont $_{K_{X^{\prime} / X}}^{e}$. Now Theorem 3.3 applies.
} 
such that all indicated maps are a blowup at a smooth center. Furthermore, there is an index $i$ such that the rational maps to $X^{\prime}$ to the left $\left(X^{\prime} \leftarrow--X_{j}\right.$ for $\left.j \leq i\right)$ and the rational maps to $X$ to the right $\left(X_{j} \rightarrow X\right.$ for $\left.j \geq i\right)$ of that index are in fact regular maps.

One should point out that the second part of the Theorem about the regularity of the maps is crucial in many of its applications, and particularly in the application that follows next.

Proof of Theorem 3.1. So far we have proved the Transformation rule for the blowup along a smooth center. Given a proper birational morphism of smooth varieties $f$ : $X^{\prime} \longrightarrow X$ we can factor it into a chain as in the Weak Factorization Theorem. In particular, for each birational map in that chain, the Transformation rule holds. The second part of the Factorzation Theorem together with the assumption that $X^{\prime} \rightarrow X$ is a morphism implies that $X_{n-1} \longrightarrow X$ is also a morphism. Part (b) of the following Exercise shows that for this morphism $X_{n-1} \longrightarrow X$ the Transformation rule holds. Now the shorter chain ending with $X_{n-2} \rightarrow X$ is again a chain such that for each map the Transformation rule holds, by part (a) of that exercise. By induction we can conclude that the transformation rule holds for $f$ itself.

Exercise 3.4. ${ }^{32}$ Suppose one is given a commuting diagram of proper birational morphisms

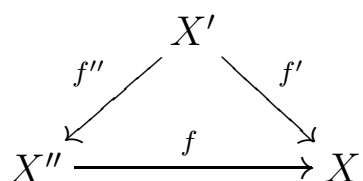

(a) If the Transformation rule holds for $f^{\prime \prime}$ and $f$, then also for $f^{\prime}=f^{\prime \prime} \circ f$.

(b) If the Transformation rule holds for $f^{\prime \prime}$ and $f^{\prime}$, then it also holds for $f$.

(c) The same as (a) and (b) but with "the Transformation rule" replaced by "the conclusions of Theorem 3.3". (This part is more difficult than the others)

(d) Using (c) and the Weak Factorization Theorem produce a proof of Theorem 3.3 building on the case of the blowup at a smooth center we considered above.

\section{BRIEF OUTLINE OF A FORMAL SETUP FOR THE MOTIVIC MEASURE.}

In this section we fill in some details that were brushed over in our treatment of motivic integration so far. First this concerns some basic properties and the welldefinedness of the motivic measure and integral.

${ }^{32}$ Solution: Part (a) is easy. Not sure if part (c) is really feasible. Part (d) is straightforward from (c). Let me outline part (b): Recall that $K_{X^{\prime} / X}=K_{X^{\prime} / X^{\prime \prime}}+f^{\prime \prime *} K_{X^{\prime \prime} / X}$ such that

$$
\begin{aligned}
& \int_{X} \mathbb{L}^{-\operatorname{ord}_{D}} d \mu_{X}=\int_{X^{\prime}} \mathbb{L}^{-\operatorname{ord}_{f^{\prime *} D-K_{X^{\prime}} / X}} d \mu_{X^{\prime}}
\end{aligned}
$$

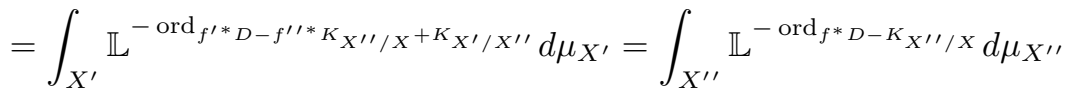

where the first and last equality is the Transformation rule for $f^{\prime \prime}$ and $f$. 
4.1. Properties of the motivic measure. For simplicity we still assume that $X$ is a smooth $\mathbb{C}$-variety. Recall that we defined for a stable set $C=\pi_{m}^{-1}(B)$ a volume by setting

$$
\mu_{X}(C)=[B] \mathbb{L}^{-n m} \in \mathcal{M}_{k}
$$

It is easy to verify that on stable sets (i.e. cylinders if $X$ is smooth) the measure is additive on finite disjoint unions. Furthermore, for stable sets $C \subseteq C^{\prime}$ one has $\operatorname{dim} \mu_{X}(C) \leq \operatorname{dim} \mu_{X}\left(C^{\prime}\right) .{ }^{33}$ We begin with a rigorous definition of what is a measurable set extending the above definition.

Definition 4.1. A subset $C \subseteq \mathcal{J}_{m}(X)$ is called measurable if for all $n \in \mathbb{N}$ there is a stable set $C_{n}$ and stable sets $D_{n, i}$ for $i \in \mathbb{N}$ such that

$$
C \Delta C_{n} \subseteq \bigcup_{i \in \mathbb{N}} D_{n, i}
$$

and $\operatorname{dim} \mu\left(D_{n, i}\right) \leq-n$ for all $i$. Here $C \Delta C_{n}=\left(C-C_{n}\right) \cup\left(C_{n}-C\right)$ denotes the symmetric difference of two sets. In this case the volume of $C$ is

$$
\mu_{X}(C)=\lim _{n \rightarrow \infty} \mu_{X}\left(C_{n}\right) \in \mathcal{M}_{k}
$$

This limit converges and is independent of the $C_{n}$ 's.

The key point in proving the claims in the definition ${ }^{34}$ is the so called Baire property of constructible subsets of a $\mathbb{C}$-variety which crucially uses the fact that $\mathbb{C}$ is uncountable, see [22, Corolaire 7.2.6].

Proposition 4.2. Let $K_{1} \supseteq K_{2} \supseteq K_{3} \supseteq \ldots$ be an infinite sequence of nonempty constructible subsets of a $\mathbb{C}$-variety $X$. Then $\bigcap_{i} K_{i}$ is nonempty.

For cylinder sets this implies the following Proposition, which will be used below in a version which asserts that a cylinder $C$ which is contained in the union of countably many cylinders $C_{i}$ is already contained in the union of finitely many of these.

Proposition 4.3. Let $C_{1} \supseteq C_{2} \supseteq C_{3} \supseteq \ldots$ be an infinite sequence of nonempty cylinders in $\mathcal{J}_{\infty}(X)$ where $X$ is a smooth $\mathbb{C}$-variety. Then $\bigcap_{i} C_{i}$ is nonempty.

Proof. By definition of a cylinder and Chevalley's theorem $\pi_{0}\left(C_{i}\right)$ is a constructible subset of $X$. Thus we can apply Proposition 4.2 to the sequence $\pi_{0}\left(C_{1}\right) \supseteq \pi_{0}\left(C_{2}\right) \supseteq \ldots$ to obtain an element $x_{0} \in \bigcap \pi_{0}\left(C_{i}\right)$. Now consider the sequence of cylinders $C_{i}^{\prime} \stackrel{\text { def }}{=}$ $C_{i} \cap \pi_{0}^{-1}\left(x_{0}\right)$ and repeat the argument for the sequence of constructible sets $\pi_{1}\left(C_{1}^{\prime}\right) \supseteq$ $\pi_{1}\left(C_{2}^{\prime}\right) \supseteq \pi_{1}\left(C_{2}^{\prime}\right) \supseteq \ldots$ to obtain an element $x_{1} \in \bigcap \pi_{1}\left(C_{i}^{\prime}\right)$. Repeating this procedure we successively lift $x_{0} \in X$ to $x_{1} \in \mathcal{J}_{1}(X), x_{2} \in \mathcal{J}_{2}(X)$ and so forth. The limit of

\footnotetext{
${ }^{33}$ Check these assertions as an exercise.

${ }^{34}$ In [33] a more restrictive definition of measurable is used, namely he requires that $\operatorname{dim} D_{n, i} \leq$ $-(n+i)$. This has the advantage that one does not require the field to be uncountable to conclude the well definedness of the measure. Essentially, he uses that if $D \subseteq \bigcup D_{i}$ are cylinders with $\lim \operatorname{dim} D_{i}=$ $-\infty$, then $D$ is already contained the union of finitely many of the $D_{i}$. This is true if $k$ is infinite, and, if $k$ is uncountable, even true without the assumption on the $D_{i}$. The advantage of Looijenga's setup is that one is not bound to an uncountable field, but unfortunatly I was not able to verify that in his setup $\mathcal{J}_{\infty}(Y)$ is measurable and has zero volume.
} 
these $x_{i}$ gives an element $x \in \mathcal{J}_{\infty}(X)$ which is in the intersection of all $C_{i}$. Thus this intersection is nonempty.

On a possibly singular $\mathbb{C}$-variety the statement (and proof) is true if "cylinder" is replaced by "stable set" above. If $X$ is a $k$-variety with $k$ at most countable these statements might be false. Nevertheless, if one views the constructible sets (resp. cylinders) not as subsets of the $k$-rational points but rather as certain sub-arragnements (something weaker than a subfunctor) of the functor of points represented by $X$ the above is essentially true. This is the point of view of Denef and Loeser and is carried out in [13].

Justification of Definition 4.1. For both claims it suffices to show $\operatorname{dim}\left(\mu_{X}\left(C_{i}\right)-\mu_{X}\left(C_{j}^{\prime}\right)\right) \leq$ $-i$ for all $j \geq i$, where the prime indicates a second set of defining data as is in the definition. We have

$$
C_{i}-C_{j}^{\prime} \subseteq\left(C \Delta C_{i}\right) \cup\left(C \Delta C_{j}^{\prime}\right) \subseteq \bigcup_{m} C_{i, m} \cup \bigcup_{m} C_{j, m}^{\prime}
$$

Since $\left(C_{i}-C_{j}^{\prime}\right)$ and all terms on the right are cylinders the previous proposition applies and $C_{i}-C_{j}^{\prime}$ is contained in finitely many of the cylinders of the right hand side. This implies that $\operatorname{dim} \mu_{X}\left(C_{i}-C_{j}^{\prime}\right) \leq-i$. The same applies to $\operatorname{dim} \mu_{X}\left(C_{j}^{\prime}-C_{i}\right)$. Using that $C_{i}=\left(C_{i} \cap C_{j}^{\prime}\right) \cup\left(C_{i}-C_{j}^{\prime}\right)$ and $C_{j}^{\prime}=\left(C_{i} \cap C_{j}^{\prime}\right) \cup\left(C_{j}^{\prime}-C_{i}\right)$ and that $\mu_{X}$ is additive on finite disjoint unions of cylinders we get

$$
\begin{aligned}
\operatorname{dim}\left(\mu_{X}\left(C_{i}\right)-\mu_{X}\left(C_{j}^{\prime}\right)\right) & =\operatorname{dim}\left(\mu_{X}\left(C_{i}-C_{j}^{\prime}\right)-\mu_{X}\left(C_{j}^{\prime}-C_{i}\right)\right) \\
& \leq \max \left\{\operatorname{dim} \mu_{X}\left(C_{i}-C_{j}^{\prime}\right), \operatorname{dim} \mu_{X}\left(C_{j}^{\prime}-C_{i}\right)\right\} \leq-i .
\end{aligned}
$$

For once this shows that the $\mu_{X}\left(C_{i}\right)$ form a Cauchy sequence, thus the limit exists as claimed. Secondly it immediately follows that this limit does not depend on the chosen data.

We summarize some basic properties of the measure and measurable sets.

Proposition 4.4. The measurable sets form an algebra of sets. $\mu_{X}$ is additive on disjoint unions, thus $\mu_{X}$ is a pre-measure in classical terminology. If $C_{i}$ are a infinite disjoint sequence of measurable sets such that

$$
\lim _{i \longrightarrow \infty} \mu_{X}\left(C_{i}\right)=0
$$

then $C=\bigcup C_{i}$ is measurable and $\mu_{X}(C)=\sum \mu_{X}\left(C_{i}\right)$.

Proof. The verification of all parts is quite easy. As an example we only show that the complement of a measurable set is also measurable and leave the rest as an exercise.

If $C$ is measurable then cylinders $C_{i}$ and $C_{i, j}$ can be chosen with the properties as in Definition 4.1. The complements $C_{i}^{c}=\mathcal{J}_{\infty}(X)-C_{i}$ are cylinders. Since $C^{c} \Delta C_{j}^{c}=$ $C \Delta C_{j}$ it follows at once that the complement of $C$ is measurable.

The following proposition was one of the missing ingredients for the setup of motivic integration we outlined so far. It ensures that our typical functions $\mathbb{L}^{-\operatorname{ord}_{Y}}$ are in fact measurable (the missing part was the level set at infinity $\mathcal{J}_{\infty}(Y)$ which we owe the proof that it is measurable with measure zero). 
Proposition 4.5. Let $Y \subseteq X$ be a locally closed subvariety. Then $\mathcal{J}_{\infty}(Y)$ is a measurable subset of $\mathcal{J}_{\infty}(X)$ and if $\operatorname{dim} Y<\operatorname{dim} X$ the volume $\mu_{X}\left(\mathcal{J}_{\infty}(Y)\right)$ is zero.

Proof. ${ }^{35}$ The proof of this result relies on a fundamental result of Greenberg [21] which, for our purpose is best phrased as follows:

Proposition 4.6. Let $Y$ be a variety. Then there exists a positive integer $c \geq 1$. such that

$$
\left.\pi_{\left\llcorner\frac{m}{c}\right.} \mathcal{J}_{\infty}(Y)=\pi_{\left\llcorner\frac{m}{c}\right.}^{m}\right\lrcorner \mathcal{J}_{m}(Y)
$$

for all $m \gg 0$.

This, in particular, implies that the image $\pi_{n}\left(\mathcal{J}_{\infty}(Y)\right)$ is a constructible subset of $\mathcal{J}_{n}(Y)$. It can be shown that its dimension is just the expected one, namely $\operatorname{dim} \pi_{n}\left(\mathcal{J}_{\infty}(Y)\right)=(n+1) \operatorname{dim} Y$, see [13, Lemma 4.3]. From these observations we obtain a bound for the dimension of $\mathcal{J}_{m}(Y)$ as follows. We can work locally and may assume that $Y \subseteq X$ for a smooth $X$. Then we have

$$
\begin{aligned}
\operatorname{dim} \mathcal{J}_{m}(Y) & \left.\leq \operatorname{dim}\left(\pi_{\left\llcorner\frac{m}{c}\right.}^{m}\right\lrcorner \mathcal{J}_{m}(Y)\right)+\left(m-\left\llcorner\frac{m}{c}\right\lrcorner\right) \operatorname{dim} X \\
& \left.=\operatorname{dim}\left(\pi_{\left\llcorner\frac{m}{c}\right.}\right\lrcorner \mathcal{J}_{\infty}(Y)\right)+\left(m-\left\llcorner\frac{m}{c}\right\lrcorner\right) \operatorname{dim} X \\
& =\left(\left\llcorner\frac{m}{c}\right\lrcorner+1\right) \operatorname{dim} Y+(m+1) \operatorname{dim} X-\left(\left\llcorner\frac{m}{c}\right\lrcorner+1\right) \operatorname{dim} X \\
& =(m+1) \operatorname{dim} X-\left(\left\llcorner\frac{m}{c}\right\lrcorner+1\right)(\operatorname{dim} X-\operatorname{dim} Y) .
\end{aligned}
$$

Thus, If the codimension of $Y$ in $X$ is greater or equal to 1 , then $\operatorname{dim} \mathcal{J}_{m}(Y) \mathbb{L}^{-m \operatorname{dim} X}$ approaches $-\infty$ as $m$ approaches $\infty$. This implies that $\mathcal{J}_{\infty}(Y)$ is measurable that its measure $\mu_{X}\left(\mathcal{J}_{\infty}(Y)\right)$ is zero.

4.1.1. Comparison with Lesbeque integration. To guide ones intuition a comparison of the motivic measure with more classical measures such as the Lesbeque measure or $p$ adic measures is sometimes helpful. We discuss here the similarities with the Lesbeque measure on $\mathbb{A}^{n}$ since this is wellknown. ${ }^{36}$

For convenience we consider the case that $X=\mathbb{A}^{n}$ in which case we identify the $k$-points of $\mathcal{J}_{\infty}\left(\mathbb{A}_{k}^{n}\right)$ with $n$-tuples of power series with coefficients in $k$. That is we identify $\mathcal{J}_{\infty}\left(\mathbb{A}_{k}^{n}\right) \cong(k \llbracket t \rrbracket)^{n}=\mathbb{A}_{k \llbracket t \rrbracket}^{n}$. Since $k \llbracket t \rrbracket$ is a discrete valuation domain (DVR) we can use the valuation to define a norm on $(k \llbracket t \rrbracket)^{n}$ by defining

$$
\|\tau\| \leq \frac{1}{m} \Longleftrightarrow \forall i: \tau_{i} \in\left(t^{m}\right) k \llbracket t \rrbracket
$$

for $\tau=\left(\tau_{1}, \ldots, \tau_{n}\right)$ a tuple of power series in $\mathcal{J}_{\infty}\left(\mathbb{A}_{k}^{n}\right)$. It is easy to check that this defines a (non-archimedian) norm. In Table 1 the similarities between Lesbeques and motivic measure are summarized.

\footnotetext{
${ }^{35}$ In [2, Proposition 6.22] Batyrev claims that with the previous results one can reduce the proof to the case that $Y$ is a smooth divisor, where it is easily verified - we discussed this on page 11 . Unfortunately I was not able to follow Batyrevs argument, thus the somewhat not so self contained proof is included here. For another another proof see Proposition 5.7.

${ }^{36}$ The analogy with $p$-adic measures is even more striking, see for example the discussions in [44] and $[24,25]$.
} 


\begin{tabular}{|c|c|c|}
\hline & Lesbeques & motivic \\
\hline space & $\mathbb{R}^{n}$ & $\mathcal{J}_{\infty}\left(\mathbb{A}^{n}\right)$ \\
values of measure & $\mathbb{Z} \subseteq \mathbb{Q} \subseteq \mathbb{R}$ & $K_{0}\left(\operatorname{Var}_{k}\right) \subseteq \mathcal{M}_{k} \subseteq \hat{\mathcal{M}}_{k}$ \\
cubes around point $a$ & $\left\{x \in \mathbb{R}^{n} \mid\|x-a\| \leq 1 / m\right\}$ & $\left\{\gamma \in \mathcal{J}_{\infty}\left(\mathbb{A}^{n}\right) \mid\|\gamma-a\| \leq 1 / m\right\}$ \\
measurable set & $\sigma$-algebra generated by cubes & algebra of stable/measurable sets \\
volume of cube & $(2 / m)^{n}$ & $\left(\mathbb{L}^{-m}\right)^{n}$ \\
transformation rule & $\int_{A} h(f) d f=\int_{g^{-1}(A)} h(f(x)) \operatorname{Jac}(f) d x$ & $\int_{A} \mathbb{L}^{-\operatorname{ord}_{D}=\int_{f_{\infty}^{-1}(A)} \mathbb{L}^{-\operatorname{ord}_{D}+K_{X^{\prime} / X}}}$ \\
\hline
\end{tabular}

TABlE 1. Comparison with Lesbeque measure.

4.2. Motivic integration on singular varieties. In the preceding discussion we used the assumption that your spaces are non-singular in several places in an essential way. Roughly speaking we used the fact that if $X$ is smooth then every cylinder is a stable set, thus can be endowed with a measure in a natural way. The fact that the resulting algebra of measurable sets to include the cylinders was essential for the setup since the level sets of the functions ord $Y$ are cylinders in a natural way.

If $X$ is singular however, many things one might got used to from the smooth case fail. Most prominently, the truncation maps are no longer surjective and a cylinder is in general not stable. Thus one has to work somewhat harder to obtain an algebra of measurable sets which include the cylinders. In order to be able to setup an integration theory one expects from this algebra of measurable sets the properties asserted in the following Proposition.

Proposition 4.7. Let $X$ be a $k$-variety. Then there is an algebra of measurable subsets of $\mathcal{J}_{\infty}(X)$ and a measure $\mu_{X}$ on that algebra such that

(1) If $A$ is stable, then $A$ is measurable and $\mu_{X}(A)=\left[\pi_{m} A\right] \mathbb{L}^{-n m}$ for $m \gg 0$.

(2) A cylinder $C$ is measurable and $\mu_{X}(C)=\lim _{m}\left[\pi_{m} C\right] \mathbb{L}^{-n m}$.

(3) The measure is additive on finite disjoint unions.

(4) If $A \subseteq B$ are measurable, then $\operatorname{dim} \mu_{X}(A) \leq \operatorname{dim} \mu_{X}(B)$.

To achieve this, one starts with the stable sets to which we know how to assign a measure. Then one proceeds just as in the smooth case using Definition 4.1, and replacing "cylinder" by "stable set" whenever necessary the same proof holds as well. The critical point now is to show that a cylinder is measurable with volume as claimed above. Even for the cylinder $\mathcal{J}_{\infty}(X)=\pi^{-1}(X)$ it is not clear a priori that it is measurable and what it's measure should be (in fact, the measure of $X$ to be constructed leads to new birational invariants of $X$ ).

The point is that one has to partition $\mathcal{J}_{\infty}(X)$ according to intersection with the singular locus Sing $X$, defined by the $n$th Fitting ideal of $\Omega_{X}^{1}$. Then we can write

$$
\mathcal{J}_{\infty}(X)=\bigsqcup_{e \geq 0} \mathcal{J}_{\infty}^{(e)}(X)
$$


where $\mathcal{J}_{\infty}^{(e)}(X)=\operatorname{ord}_{\operatorname{Sing} X}^{-1}(e)$. As it turns out, the sets $\mathcal{J}_{\infty}^{(e)}(X)$ are in fact stable at level $\geq e$. The method is analogous to partitioning the cylinders of $X^{\prime}$ according to intersection with $K_{X^{\prime} / X}$ (defined by the 0th Fitting ideal of $\omega_{X^{\prime} / X}$ ) in the proof of the transformation rule. If one treats everything subordinate to this partition according to intersection with the singular locus one can construct a working theory in the singular case, see $[33,12]$.

\section{Birational INVARIANTS VIA MOtiViC INTEGRATION}

As an illustration of the theory we discuss some applications of geometric motivic integration to birational geometry, namely we give a description of the log canonical threshold of a pair $(X, Y)$, where $Y$ is a closed subscheme of the smooth scheme $X$, in terms of the asymptotic behavior of the dimensions of the jet schemes $\mathcal{J}_{m}(Y)$. These results are due to Mustaţă [36] and his collaborators [15, 17, 45]. The precise statement is as follows:

Theorem 5.1. Let $Y \subseteq X$ be a subscheme of the smooth variety $X$. Then the $\log$ canonical threshold of the pair $(X, Y)$ is

$$
c(X, Y)=\operatorname{dim} X-\sup _{m}\left\{\frac{\operatorname{dim} \mathcal{J}_{m}(Y)}{m+1}\right\} .
$$

The definition of the log canonical threshold requires the introduction of some more notation from birational geometry which will be done shortly. It is an invariant which can be read of from the data of a log resolution of the pair $(X, Y)$. The proof of the above is a very typical application of motivic integration as its strategy is to express the quantity one is interested in ( $\operatorname{say} \operatorname{dim} \mathcal{J}_{m}(Y)$ ), in terms of a motivic integral. Then one uses the transformation rule (Theorem 3.1) to reduce to an integral over a normal crossing divisor which can be explicitly computed, similarly as Formula 2.6.

This result is in line with the earliest investigations of jet spaces by Nash [37] who conjectured an intimate correspondence between the jet spaces of a singular space and the divisors appearing in a resolution of singularities. Even though his conjecture was disproved recently by Kollar and Ishii [27] in general, there are important cases where his prediction was true, for example in the case of toric varieties.

5.1. Notation from birational geometry. Throughout this section we fix the following setup. Let $X$ be a smooth $k$-variety of dimension $n$ and let $k$ be of characteristic zero. Let $Y \subseteq X$ be a closed subscheme. Let $f: X^{\prime} \rightarrow X$ be a log resolution of the pair $(X, Y)$, that is a proper birational map such that

(1) $X^{\prime}$ is smooth and

(2) denoting by $F \stackrel{\text { def }}{=} f^{-1} Y=\sum_{i=1}^{s} a_{i} D$ and $K \stackrel{\text { def }}{=} K_{X^{\prime} / X}=\sum_{i=1}^{s} b_{i} D$ for some $a_{i}, b_{i} \in \mathbb{Q}$ and prime divisors $D_{i}$, the divisors $F, K$ and $F+K$ have simple normal crossing support.

The existence of log resolutions is a consequence of Hironaka's resolution of singularities. Now one defines: 
Definition 5.2. Let $X$ and $Y$ and the datum of a log resolution be as above and let $q \geq 0$ be a rational number. Then we say that

(1) $(X, q \cdot Y)$ is Kawamata log terminal (KLT) if and only if $b_{i}-q a_{i}+1>0$ for all $i$.

(2) $(X, q \cdot Y)$ is $\log$ canonical (LC) if and only if $b_{i}-q a_{i}+1 \geq 0$ for all $i$.

We point out (without proof) that these notions are independent of the chosen log resolution and therefore well defined, see [32] for details.

Remark 5.3. These notions can be expressed in terms of the multiplier ideal $\mathcal{I}\left(q \cdot I_{Y}\right)$ of $I_{Y}$, the sheaf of ideals which cuts out $Y$ on $X$, as follows:

$$
\begin{aligned}
(X, q \cdot Y) \text { is } \mathrm{KLT} & \Longleftrightarrow \mathcal{I}\left(I_{Y}^{q}\right)=\mathcal{O}_{X} \\
(X, q \cdot Y) \text { is LC } & \Longleftrightarrow \mathcal{I}\left(I_{Y}^{q}\right)=\mathcal{O}_{X} \quad \forall q^{\prime}<q .
\end{aligned}
$$

To see this observe that by definition

$$
\mathcal{I}\left(I_{Y}^{q}\right)=f_{*} \mathcal{O}_{X^{\prime}}(\ulcorner K-q F\urcorner)=f_{*} \mathcal{O}_{X^{\prime}}\left(\left\ulcorner b_{i}-q a_{i}\right\urcorner D_{i}\right)
$$

which is equal to $\mathcal{O}_{X}$ if and only if $\left\ulcorner b_{i}-q a_{i}\right\urcorner \geq 0$ for all $i$. as the upper corners denote the round up of an integer, this is equivalent to $b_{i}-q a_{i}+1>0$ for all $i$ as required.

Now we proceed to the definition of the log canonical threshold, which is just the largest $q$ such that the pair $(X, q \cdot Y)$ is Kawamata log terminal.

Definition 5.4. The log canonical threshold of the pair $(X, Y)$ is

$$
\begin{aligned}
\operatorname{lct}(X, Y) & =\sup \{q \mid(X, q \cdot Y) \text { is } \operatorname{KLT}\} \\
& =\sup \left\{q \mid b_{i}-q a_{i}+1>0 \quad \forall i\right\} \\
& =\min _{i}\left\{\frac{b_{i}+1}{a_{i}}\right\}
\end{aligned}
$$

Note that clearly one has $\operatorname{lct}(X, q \cdot Y)=q^{-1} \operatorname{lct}(X, Y)$ so that we can restrict to the case $q=1$ in the definition of the $\log$ canonical threshold. The formula for the log canonical threshold in terms of the jet schemes which we are aiming to proof in this section is an immediate consequence of the following theorem.

Theorem 5.5. Let $X$ and $Y$ be as before. Then

$$
\begin{aligned}
(X, q \cdot Y) \text { is } K L T & \Longleftrightarrow \operatorname{dim} \mathcal{J}_{m}(Y)<(m+1)(n-q) \text { for all } m \\
(X, q \cdot Y) \text { is } L C & \Longleftrightarrow \operatorname{dim} \mathcal{J}_{m}(Y) \leq(m+1)(n-q) \text { for all } m .
\end{aligned}
$$

From this the proof of Theorem 5.1 follows immediately.

Proof of Theorem 5.1. By definition we have

$$
\begin{aligned}
\operatorname{lct}(X, Y) & =\sup \{q \mid(X, q \cdot Y) \text { is KLT }\} \\
& =\sup \left\{q \mid \operatorname{dim} \mathcal{J}_{m}(Y)<(m+1)(n-q) \forall m\right\} \\
& =n-\sup _{m}\left\{\frac{\operatorname{dim} \mathcal{J}_{m}(Y)}{m+1}\right\}
\end{aligned}
$$


Moreover, the proof of Theorem 5.5 will reveal that the supremum in Theorem 5.1 is actually obtained by infinitely many $m$, namely whenever $m+1$ is divisible by all the coefficients $a_{i}$ of $D_{i}$ in $f^{-1} Y=\sum a_{i} D_{i}$ one has $\operatorname{lct}(X, Y)=\operatorname{dim} X-\frac{\operatorname{dim} \mathcal{J}_{m}(Y)}{m+1}$.

Before proceeding to the proof we derive some elementary properties of the log canonical threshold.

Proposition 5.6. Let $Y \subseteq Y^{\prime} \subseteq X$ closed subvarieties of $X$. Then

(1) $\operatorname{lct}(X, Y) \geq \operatorname{lct}\left(X, Y^{\prime}\right)$.

(2) $0<\operatorname{lct}(X, Y) \leq \operatorname{codim}(Y, X)$ with equality if $(X, Y)$ is $\log$ canonical.

(3) $\operatorname{lct}(X, Y)$ is independent of $X$ of fixed dimension.

(4) Let $\left(X^{\prime}, Y^{\prime}\right)$ be another pair, then $\operatorname{lct}\left(X \times X^{\prime}, Y \times Y^{\prime}\right)=\operatorname{lct}(X, Y)+\operatorname{lct}\left(X^{\prime}, Y^{\prime}\right)$.

Proof. For (1) note that $Y \subseteq Y^{\prime}$ implies that $\operatorname{dim} \mathcal{J}_{m}(Y) \leq \operatorname{dim} \mathcal{J}_{m}\left(Y^{\prime}\right)$ then apply Theorem 5.1.

For (2) recall that in any case $\mathcal{J}_{m}(Y)$ contains $\mathcal{J}_{m}\left(Y_{\text {reg }}\right)$ the jet scheme over the regular locus of $Y$. The latter has dimension $(m+1) \operatorname{dim} Y$. Therefore $\operatorname{dim} \mathcal{J}_{m}(Y) \geq$ $(m+1) \operatorname{dim} Y$ and we finish by applying Theorem 5.1.

(3) is immediate since in the formula for $\operatorname{lct}(X, Y)$ the only feature of $X$ that appears is its dimension.

The formation of jet schemes preserves products ${ }^{37}$ and hence it follows that $\operatorname{dim} \mathcal{J}_{m}(Y \times$ $\left.Y^{\prime}\right)=\operatorname{dim} \mathcal{J}_{m}(Y)+\operatorname{dim} \mathcal{J}_{m}\left(Y^{\prime}\right)$ from which (4) is implied immediately.

5.2. Proof of threshold formula. Now we present the proof of Theorem 5.5. For this we first recall the transformation rule in a slightly more general form than stated above. Let $A \subseteq \mathcal{J}_{\infty}(X)$ be a measurable subset (for example a stable subset) and let $g: \mathcal{J}_{\infty}(X) \longrightarrow \mathbb{Q}$ be a function whose level sets are measurable. Then

$$
\int_{A} \mathbb{L}^{g} d \mu_{X}=\int_{f_{\infty}^{-1}(A)} \mathbb{L}^{g \circ f_{\infty}-\operatorname{ord}_{K_{X^{\prime}} / X}} d \mu_{X^{\prime}}
$$

provided that one of the integrals exists, which then implies the existence of the other. The new features are minor. Clearly, it is allowed to integrate only over a measurable subset $A$ as long as we also only integrate over its measurable image $f^{\infty}(A)$ as well (that this image is measurable can be deduced from the proof of the transformation rule). In order to make the expression $\mathbb{L}^{q}$ for rational $q$ defined we have to adjoin roots of $\mathbb{L}$ to the already huge ring $\mathcal{M}_{k}$ and define the dimension of $\mathbb{L}^{q}$ as $q$. For the following application it is in fact enough to adjoin a single root of $\mathbb{L}$ such that the new value ring of the integral is $\hat{\mathcal{M}}_{k}\left[\mathbb{L}^{1 / n}\right]$ for a sufficiently big $n$.

We apply this result with $g=q \cdot \operatorname{ord}_{Y}=\operatorname{ord}_{q Y}$ and $A=\operatorname{ord}_{Y}^{-1}(m+1)$ so that $f_{\infty}^{-1}(A)=\operatorname{ord}_{F}^{-1}(m+1)$ (up to measure zero) and $g \circ f_{\infty}-\operatorname{ord}_{K_{X^{\prime} / X}}=-\operatorname{ord}_{K_{X^{\prime} / X}-q F}$. Thus we get:

$$
\int_{\operatorname{ord}_{Y}^{-1}(m+1)} \mathbb{L}^{q \operatorname{ord}_{Y}} d \mu_{X}=\int_{\operatorname{ord}_{F}^{-1}(m+1)} \mathbb{L}^{-\operatorname{ord}_{X_{X^{\prime}} / X^{-q F}}} d \mu_{X^{\prime}}
$$

Now, the left hand side of this equation contains information about the dimension of the $m$ th jet scheme $J_{m}(Y)$, whereas the right hand side allows us to express this

\footnotetext{
${ }^{37}$ By definition the functor $\mathcal{J}_{m}\left(\_\right)$has a left adjoint thus commutes with direct products.
} 
dimension in terms of the data of the log resolution. Together this will lead to a proof of Theorem 5.5.

Recall from Section 2.4 that

$$
\operatorname{ord}_{Y}^{-1}(m+1)=\pi_{m}^{-1}\left(\mathcal{J}_{m}(Y)\right)-\pi_{m+1}^{-1}\left(\mathcal{J}_{m+1}(Y)\right)
$$

and thus for the measure we have

$$
\mu_{X}\left(\operatorname{ord}_{Y}^{-1}(m+1)\right)=\left[\mathcal{J}_{m}(Y)\right] \mathbb{L}^{-n m}-\left[\mathcal{J}_{m+1}(Y)\right] \mathbb{L}^{-n(m+1)} .
$$

Now computing the left hand side of equation (5) one gets

$$
\begin{aligned}
S_{m} & \stackrel{\text { def }}{=} \int_{\operatorname{ord}_{Y}(m+1)} \mathbb{L}^{q \operatorname{ord}_{Y}} d \mu_{X}=\mu_{X}\left(\operatorname{ord}_{Y}^{-1}(m+1)\right) \mathbb{L}^{q(m+1)} \\
& =\left(\left[\mathcal{J}_{m}(Y)\right]-\left[\mathcal{J}_{m+1}(Y)\right] \mathbb{L}^{-n}\right) \mathbb{L}^{-n m+q(m+1)}
\end{aligned}
$$

Recalling that

$$
\operatorname{dim}\left[\mathcal{J}_{m}(Y)\right]+n \geq \operatorname{dim}\left[\mathcal{J}_{m+1}(Y)\right]
$$

this implies, while evaluating at the dimension (see section 2.1), that

$$
\operatorname{dim} S_{m} \leq \operatorname{dim} \mathcal{J}_{m}(Y)-n m+q(m+1)
$$

with " $<$ " holding only if we have equality in (6). This is a consequence of the property of the dimension function which says that $\operatorname{dim}(A+B) \leq \max \{\operatorname{dim} A, \operatorname{dim} B\}$ with equality as soon as $\operatorname{dim} A \neq \operatorname{dim} B$.

Now we turn to computing the right hand side of equation (5):

$$
\begin{aligned}
\int_{\operatorname{ord}_{F}^{-1}(m+1)} \mathbb{L}^{-\operatorname{ord}_{K-q F} d \mu_{X^{\prime}}} & =\sum_{i=1}^{\infty} \mu_{X^{\prime}}\left(\operatorname{ord}_{F}^{-1}(m+1) \cap \operatorname{ord}_{K-q F}^{-1}(i)\right) \mathbb{L}^{-i} \\
& =\sum_{r \in A_{m}} \mu_{X^{\prime}}\left(\bigcap_{i} \operatorname{ord}_{D_{i}}^{-1}\left(r_{i}\right)\right) \mathbb{L}^{-\sum\left(b_{i}-q a_{i}\right) r_{i}}
\end{aligned}
$$

where the last equality relies on a partitioning of $\operatorname{ord}_{F}^{-1}(m+1)$, according to intersection with each component $D_{i}$ of the occurring normal crossing divisors:

$$
\begin{gathered}
A_{m}=\left\{r=\left(r_{1}, \ldots, r_{s}\right) \mid r_{i} \geq 0, \sum a_{i} r_{i}=m+1\right\} \quad\left(\text { this ensures } \operatorname{ord}_{F}=m+1\right) \\
\operatorname{ord}_{F}^{-1}(m+1)=\bigsqcup_{r \in A_{m}}\left(\bigcap_{i} \operatorname{ord}_{D_{i}}^{-1}\left(r_{i}\right)\right)
\end{gathered}
$$

Clearly, this refines the partition $\operatorname{ord}_{F}^{-1}(m+1)=\bigsqcup_{i}\left(\operatorname{ord}_{F}^{-1}(m+1) \cap \operatorname{ord}_{K-q F}^{-1}(i)\right)$ and we have for $\gamma \in \cap \operatorname{ord}_{D_{i}}^{-1}\left(r_{i}\right)$ that $\operatorname{ord}_{K-q F}(\gamma)=\sum\left(b_{i}-q a_{i}\right) r_{i}$ which justifies the above computation. The point now is that $\mu_{X^{\prime}}\left(\bigcap_{i} \operatorname{ord}_{D_{i}}^{-1}\left(r_{i}\right)\right)$ was computed explicitly in Lemma 2.7 to be equal to $\left[D_{\text {supp } r}^{0}\right](\mathbb{L}-1)^{|\operatorname{supp} r|} \mathbb{L}^{-\sum r_{i}}$. Hence

$$
\begin{aligned}
S_{m} & =\int_{\operatorname{ord}_{F}^{-1}(m+1)} \mathbb{L}^{-\operatorname{ord}_{K-q F}} d \mu_{X^{\prime}} \\
& =\sum_{r \in A_{m}}\left[D_{\operatorname{supp} r}^{0}\right](\mathbb{L}-1)^{|\operatorname{supp} r|} \mathbb{L}^{-\sum\left(b_{i}-q a_{i}+1\right) r_{i}}
\end{aligned}
$$


Note that the dimension of each summand is equal to $n-|\operatorname{supp} r|+|\operatorname{supp} r|-\sum\left(b_{i}-\right.$ $\left.q a_{i}+1\right) r_{i}=n-\sum\left(b_{i}-q a_{i}+1\right) r_{i}$, and since the coefficient of the highest dimensional part of each summand has a positive sign, there is no cancellation of highest dimensional parts in the sum. Thus we get

$$
\operatorname{dim} S_{m}=\max _{r \in A_{m}}\left\{n-\sum\left(b_{i}-q a_{i}+1\right) r_{i}\right\}
$$

With the formulas (A) and (B) at hand the proof of Theorem 5.5 follows easily.

Proof of Theorem 5.5. It is enough to prove the first equivalence of Theorem 5.5, the second one being a limiting case of the first. Slightly reformulating and using the definition of KLT we have to show that

$$
b_{i}-q a_{i}+1>0 \quad \forall i \Longleftrightarrow \operatorname{dim} \mathcal{J}_{m}(Y)-n m+q(m+1)<n \quad \forall m
$$

Let us first treat the implication " $\Leftarrow$ ": In fact, we only need to assume the right hand side for one $m_{0}$ such that $m_{0}+1$ is divisible by each $a_{i}$. Then using equation (A) we have $\operatorname{dim} S_{m_{0}} \leq \operatorname{dim} \mathcal{J}_{m_{0}}(Y)-n m_{0}+q\left(m_{0}+1\right)<n$. Since $b_{i}-q a_{i}+1>0$ holds trivially for $a_{i}=0$ we only need to consider $i$ such that $a_{i} \neq 0$. In this case (for fixed $i$ ) define the tuple $r=\left(r_{1}, \ldots, r_{s}\right)$ by setting $r_{i}=\frac{m_{0}+1}{a_{i}}$ and $r_{j}=0$ otherwise. Clearly $r \in A_{m_{0}}$. Now, equation (B) implies

$$
n>\operatorname{dim} S_{m_{0}} \geq n-\sum_{j=1}^{s}\left(b_{j}-q a_{j}+1\right) r_{j}=n-\left(b_{i}-q a_{i}+1\right) r_{i}
$$

which says nothing but that $b_{i}-q a_{i}+1>0$ as required.

Now we treat the converse " $\Rightarrow$ ": Assuming that the pair $(X, q \cdot Y)$ is $\operatorname{KLT}$ (i.e. $\operatorname{dim} S_{m}<n$ for all $m$ by equation (B)) and that

$$
\operatorname{dim} \mathcal{J}_{m}(Y)-n m+q(m+1) \geq n
$$

for some $m$ we seek a contradiction. These two assumptions together imply that the inequality (A) is strict, that is we have $\operatorname{dim} S_{m}<\operatorname{dim} \mathcal{J}_{m}(Y)-n m+q((m+1)$. This, as we argued before, happens only if

$$
\operatorname{dim} \mathcal{J}_{m}(Y)=\operatorname{dim} \mathcal{J}_{m+1}(Y)-n .
$$

Substituting this last equality into (7) we get (7) for $m$ replaced by $m+1$. Repeating this we obtain

$$
\operatorname{dim} \mathcal{J}_{m+i}(Y)=\operatorname{dim} \mathcal{J}_{m}(Y)+i n
$$

for all $i$ which contradicts Proposition 5.7 below.

We include here a more elementary and more explicit version of 4.5. This is also due to Mustaţă, we only sketch the proof here and refer the reader to [35, Lemma 3.7].

Proposition 5.7. Let $X$ be smooth of dimension $n$ and let $Y \subseteq X$ be a subvariety. Let $a=$ mult $_{y} Y$ be the local multiplicity of $Y$ at the point $y \in Y$, then

$$
\operatorname{dim}\left(\pi_{Y}^{m}\right)^{-1}(y) \leq m \cdot \operatorname{dim} X-\left\llcorner\frac{m}{a}\right\lrcorner .
$$

Thus if a is now the maximum of all local multiplicities of $Y$ one has

$$
\operatorname{dim} \mathcal{J}_{m}(Y) \leq \operatorname{dim} Y+m \cdot \operatorname{dim} X-\left\llcorner\frac{m}{a}\right\lrcorner \leq(m+1) \cdot \operatorname{dim} X-\left\llcorner\frac{m}{a}\right\lrcorner .
$$


It follows that if $Y$ is nowhere dense in $X$, then $\mu_{X}\left(\mathcal{J}_{\infty}(Y)\right)=0$.

Proof. The second statement clearly follows from the first which in turn immediately reduces to the case that $Y \subseteq X$ is a hypersurface. Since $X$ is smooth it is étale over $\mathbb{A}^{n}$ with $y$ mapping to 0 . Since $\left(\pi_{X}^{\infty}\right)^{-1}(y)$ gets thereby identified with $\left(\pi_{\mathbb{A}^{n}}^{\infty}\right)^{-1}(0)$ we may assume that $Y \subseteq \mathbb{A}^{n}$ is given by the vanishing of $f \in k[x]=k\left[x_{1}, \ldots, x_{n}\right]$ and the point $y$ is the origin.

The condition that the local multiplicity of $Y$ at 0 is equal to $a$ means that the smallest degree monomial of $f$ has degree $a$ in $x_{0}, \ldots, x_{n}$. For simplicity we assume now that $f$ is homogeneous of degree $a$, in general one can combine the following proof with a deformation argument to reduce to this case along the way, see [35] for this general case.

Exercise 2.5 states that $\mathcal{J}_{m}(Y) \subseteq \mathcal{J}_{m}\left(\mathbb{A}^{n}\right)$ is given by $m+1$ equations $f^{(0)}, \ldots, f^{(m)}$ in the coordinates of $\mathcal{J}_{m}\left(\mathbb{A}^{n}\right)$ described in Example 2.1. Concretely, $f^{(i)} \in k\left[x^{(0)}, \ldots, x^{(i)}\right]$ is given as the coefficient of $t^{i}$ in the power series

$$
f\left(\sum_{i} x_{1}^{(i)} t^{i}, \ldots, \sum_{i} x_{n}^{(i)} t^{i}\right) .
$$

With the notation $f_{0}^{(i)} \stackrel{\text { def }}{=} f^{(i)}\left(0, x^{(1)}, x^{(2)}, \ldots, x^{(i)}\right)$ (we abbreviated the tuples $x_{1}^{(i)}, \ldots, x_{n}^{(i)}$ by $\left.x^{(i)}\right)$ the fiber $\left(\pi_{Y}^{m}\right)^{-1}(0)$ is given by the vanishing of $f_{0}^{(1)}, \ldots, f_{0}^{(m)}$ in the fiber $\left(\pi_{X}^{m}\right)^{-1}(0) \cong \operatorname{Spec} k\left[x^{(1)}, \ldots, x^{(m)}\right]$. Recall that we need to show that the dimension of $\left(\pi_{Y}^{m}\right)^{-1}(0)$ is at most $m n-\left\llcorner\frac{m}{a}\right\lrcorner$. Thus it is enough to show that the dimension of the variety given by the vanishing of the ideal $I_{p}=\left(f_{0}^{(a)}, f_{0}^{(2 a)}, \ldots, f_{0}^{(p a)}\right)$ is at most (pa) $n-p$.

To show this we turn to an initial ideal of $I_{p}$ with respect to the degree reverse lexicographic order on $k\left[x^{(1)}, \ldots, x^{(p a)}\right]$ where the underlying ordering of the variables $x_{i}^{(j)}$ is first by upper index and then by lower. ${ }^{38}$ Now it is a matter of unravelling the definitions $^{39}$ (of the $f^{(i)}$, of the order ...) to see that

$$
\operatorname{in}_{<}\left(f_{0}^{(a j)}\right)=\operatorname{in}_{<}(f)\left(x^{(j)}\right) \text { for } j=1, \ldots, p .
$$

As these are $p$ many nontrivial equations in disjoint variables it follows that the dimension of the vanishing locus of the initial ideal of $I_{p}$ is at most $(p a) n-p$. Thus the same upper bound holds for the dimension of the vanishing locus of $I_{p}$ itself, and a forteriori for the dimension of $\left(\pi_{Y}^{p a}\right)^{-1}(0)$.

${ }^{38}$ That is we order the variables according to $x_{i}^{(j)} \leq x_{i^{\prime}}^{\left(j^{\prime}\right)}$ iff $j>j^{\prime}$ or $j=j^{\prime}$ and $i<i^{\prime}$. The degree reverse lexicographic order on a polynomial ring $k\left[x_{1}, \ldots, x_{n}\right]$ with $x_{1}>x_{2}>\ldots>x_{n}$ is given by

$$
A=x_{1}^{a_{1}} \cdots x_{n}^{a_{n}}>x_{1}^{b_{1}} \cdots x_{n}^{b_{n}}=B
$$

if $\operatorname{deg} A>\operatorname{deg} B$ or $\operatorname{deg} A=\operatorname{deg} B$ and $a_{i}<b_{i}$ for the last index $k$ for which $a_{i} \neq b_{i}$. Roughly speaking, a monomial in degree rev lex is big if it contains fewest of the cheap variables. For example $x_{2}^{4}>x_{1}^{3} x_{3}>x_{1}^{2} x_{2} x_{3}$. Consult [18, Chapter 15] for details.

${ }^{39}$ Show that for two monomials $A<B$ in $k[x]$ one has $\operatorname{in}_{<}\left(A_{0}^{(j)}\right)<\operatorname{in}_{<}\left(B_{0}^{(j)}\right)$. This reduces to the case that $f=\operatorname{in}_{<}(f)$ is a monomial. Then observe that the monomial $f\left(x^{(j)}\right)$ appears in $f^{(j a)}$. Since the rev-lex-ordering is such that a term is large if it contains fewest variables which are small it follows that $f\left(x^{(j)}\right)$ is in fact the leading term as claimed. 
5.3. Bounds for the log canonical threshold. In this section we show how the just derived description of the log canonical threshold in terms of the dimension of the jet spaces lead to some interesting bounds for $\operatorname{lct}(X, Y)$.

Proposition 5.8. Let a be the maximal local multiplicity of a point in $Y$. Then

$$
\frac{1}{a} \leq \operatorname{lct}(X, Y) \leq \frac{\operatorname{dim} X}{a} .
$$

Proof. We may assume that $Y$ is neither $\emptyset$ nor all of $X$ since these cases are trivial. Let $p$ be a point with maximal multiplicity $a$. The second part of Exercise 5.1 shows that $\operatorname{dim} \mathcal{J}_{a-1}(Y) \geq \operatorname{dim}\left(\pi^{a-1}\right)^{-1}(p) \geq \operatorname{dim} X \cdot(a-1)$. Hence by Theorem 5.1

$$
\operatorname{lct}(X, Y) \leq \operatorname{dim} X-\frac{\operatorname{dim} \mathcal{J}_{a-1}(Y)}{a} \leq \operatorname{dim} X-\operatorname{dim} X \frac{a-1}{a}=\frac{\operatorname{dim} X}{a}
$$

For the lower bound Proposition 5.7 gives $\operatorname{dim} \mathcal{J}_{m}(Y) \leq \operatorname{dim} Y+m \cdot \operatorname{dim} X-\left\llcorner\frac{m}{a}\right\lrcorner$ and again using Theorem 5.1 this yields

$$
\operatorname{lct}(X, Y) \geq \frac{\operatorname{dim} X-\operatorname{dim} Y}{m+1}+\frac{\left\llcorner\frac{m}{a}\right\lrcorner}{m+1}
$$

for all $m$. For sufficiently divisible and sufficiently big $m$ this implies that $\operatorname{lct}(X, Y) \geq \frac{1}{a}$ as claimed.

The next application of the arc space techniques is a bound for the log canonical threshold of a homogeneous hypersurface. This uses the following exercise as a key ingredient.

Exercise 5.1. ${ }^{40}$ Let $Y \subseteq \mathbb{A}^{n}$ be a homogeneous hypersurface of degree $d$. Show that one has an isomorphism

$$
\left(\pi_{Y}^{m}\right)^{-1}(0) \cong \mathcal{J}_{m-d}(Y) \times \mathbb{A}^{n(d-1)}
$$

for all $m \geq d-1$, where we set $\mathcal{J}_{-1}(Y)$ to be a point.

Drop the assumption homogeneous and assume instead that the local multiplicity of $Y$ at $p$ be equal to $a$. Show that $\left(\pi_{Y}^{a-1}\right)^{-1}(p) \cong \mathbb{A}^{n(a-1)}$.

Proposition 5.9. Let $Y \subseteq \mathbb{A}^{n}$ be a homogeneous hypersurface of degree $d$. Then

$$
\operatorname{lct}\left(\mathbb{A}^{n}, Y\right) \geq \min \left\{\frac{n-r}{d}, 1\right\}
$$

where $r=\operatorname{dim} \operatorname{Sing} Y$.

Proof. One key ingredient is the observation of the previous Exercise 5.1 that for $m \geq$ $d-1$

$$
\left(\pi_{Y}^{m}\right)^{-1}(0) \cong \mathcal{J}_{m-d}(Y) \times \mathbb{A}^{n(d-1)}
$$

By semicontinuity of $\operatorname{dim}\left(\pi_{Y}^{m}\right)^{-1}(p)$ the inequality

$$
\operatorname{dim}\left(\pi_{Y}^{m}\right)^{-1}(p) \leq \operatorname{dim} \mathcal{J}_{m-d}(Y)+n(d-1)
$$

\footnotetext{
${ }^{40}$ This exercise is solved by explicitly writing down the equations which define $\left(\pi_{Y}^{m}\right)^{-1}(0)$ within $\left(\pi_{\mathbb{A}^{n}}^{m}\right)^{-1}(0)$. These turn out to be the same as the defining equations of the right hand side; just the variables are different.
} 
holds for all $p$, and in particular for the $p \in \operatorname{Sing} Y$. Hence all together we get the estimate

$$
\operatorname{dim} \mathcal{J}_{m}(Y) \leq \max \left\{\operatorname{dim} \mathcal{J}_{m-d}(Y)+n(d-1),(n-1)(m+1)\right\}
$$

where $(n-1)(m+1)$ is equal to $\operatorname{dim} \mathcal{J}_{m}(Y-\operatorname{Sing} Y)$ which is always a lower bound for $\operatorname{dim} \mathcal{J}_{m}(Y)$. Now set $m=p d-1$ and apply the inequality repeatedly to get

$$
\operatorname{dim} \mathcal{J}_{p d-1}(Y) \leq \max \{(n d-n+r) \cdot p,(n-1) p d\}
$$

which amounts to

$$
\operatorname{lct}\left(\mathbb{A}^{n}, Y\right)=n-\frac{\operatorname{dim} \mathcal{J}_{p d-1}(Y)}{p d} \geq \min \left\{\frac{n-r}{d}, 1\right\}
$$

since the log canonical threshold is computed via the dimension of the jet spaces $\mathcal{J}_{p d-1}(Y)$ for sufficiently divisible $p d$.

In [15] the main achievement is to characterize the extremal case as follows: In the case that $\operatorname{lct}\left(\mathbb{A}^{n}, Y\right) \neq 1$ one has $\operatorname{lct}\left(\mathbb{A}^{n}, Y\right)=\frac{n-r}{d}$ if and only if $Y \cong Y^{\prime} \times \mathbb{A}^{r}$ for some $Y^{\prime} \subseteq \mathbb{A}^{n-r}$ a hypersurface.

5.4. Inversion of adjunction. One of the most celebrated applications of motivic integration to birational geometry is a much improved understanding of the Inversion of Adjunction conjecture of Shokurov [42] and Kollar [29]. The conjecture describes how certain invariants of singularities of pairs behave under restriction. The following proposition goes in this direction as it shows that under restriction to a smooth hypersurface the log canonical threshold can only decrease, that is the singularities cannot get better under restriction.

Proposition 5.10. Let $(X, Y)$ be a pair and $H$ a smooth hypersurface in $X$. Then

$$
c_{H}(X, Y) \geq \operatorname{lct}(H, Y \cap H)
$$

where $c_{H}(X, Y)$ is the log canonical threshold of the pair $(X, Y)$ around $H$, that is the minimum $\operatorname{lct}(U, Y \cap U)$ over all open $U \subseteq X$ with $U \cap H \neq \emptyset$.

Proof. The proof uses a straightforward extension of the formula for the log canonical threshold to include this more general case of $c_{H}(X, Y)$. In fact the same proof as above shows that one has

$$
c_{H}(X, Y)=\operatorname{dim} X-\sup _{m}\left\{\frac{\operatorname{dim}_{H} \mathcal{J}_{m}(Y)}{m+1}\right\}
$$

with equality for sufficiently divisible $m+1$. Here $\operatorname{dim}_{H} \mathcal{J}_{m}(Y)$ is the dimension of $\mathcal{J}_{m}(Y)$ along $H$, that is the maximal dimension of an irreducible component $T$ of $\mathcal{J}_{m}(Y)$ such that $\pi^{m}(T) \cap H \neq \emptyset$.

Let $T$ be an irreducible component of $\mathcal{J}_{m}(Y)$ such that $\pi^{m}(T) \cap H \neq \emptyset$. This implies that $T \cap \mathcal{J}_{m}(Y \cap H)$ is also nonempty since the projection $\pi_{Y \cap H}^{m}$ is surjective. Since $H \subseteq X$ is locally given by one equation the same is true for $H \cap Y \subseteq Y$. Hence 
$\mathcal{J}_{m}(H \cap Y) \subseteq \mathcal{J}_{m}(Y)$ is given by at most $m+1$ equations (see Exercise 2.5). Hence $\operatorname{dim}_{H} \mathcal{J}_{m}(Y) \leq \operatorname{dim} \mathcal{J}_{m}(Y \cap H)+(m+1)$ which shows

$$
\sup _{m}\left\{\frac{\operatorname{dim}_{H} \mathcal{J}_{m}(Y)}{m+1}\right\} \leq \sup _{m}\left\{\frac{\operatorname{dim}_{H} \mathcal{J}_{m}(Y \cap H)}{m+1}\right\}+1
$$

which implies the claimed inequality $c_{H}(X, Y) \geq \operatorname{lct}(H, Y \cap H)$.

The Inversion of Adjunction Conjecture of Kollár and Shokurov describes how the singularities of pair behave under restriction to a Cartier divisor. More precisely, let $(X, Y)$ be a pair where we allow $Y=\sum q_{i} Y_{i}$ to be any formal integer combination (rational or real combination even) of closed subvarieties of $X$. With the notation $f: X^{\prime} \longrightarrow X$ of a log resolution as above (in particular $f^{-1} Y=\sum a_{i} E_{i}$ and $K_{X^{\prime} / X}=$ $\left.\sum b_{i} E_{i}\right)$ and a subvariety $W \subseteq X$ fixed we define the minimal log discrepancy

$$
\operatorname{mld}(W ; X, Y) \stackrel{\text { def }}{=} \begin{cases}\min \left\{b_{i}-a_{i}+1 \mid f\left(E_{i}\right) \subseteq W\right\} & \text { if this minimum is non-negative } \\ -\infty & \text { otherwise. }\end{cases}
$$

It follows that $(X, Y)$ is $\log$ canonical on an open subset containing $W$ iff $\operatorname{mld}(W ; X, Y) \neq$ $-\infty$. The inversion of adjunction conjecture now states:

Conjecture 5.11. With $(X, Y)$ and as above, let $D$ be a normal effective Cartier divisor on $X$ such that $D \nsubseteq Y$ and let $W \subset D$ a proper closed subset. Then we have

$$
\operatorname{mld}(W ; X, Y+D)=\operatorname{mld}\left(W ; D,\left.Y\right|_{D}\right) .
$$

The inequality " $\leq$ " is the adjunction part and is well known to follow from the adjunction formula $K_{D}=\left.\left(K_{X}+D\right)\right|_{D}$. The reverse inequality " $\geq$ " is the critical part of this conjecture.

In [17] the conjecture was proved in the case that $X$ is smooth and $Y$ is effective. In [16] this was established even for $X$ a complete intersection (and $Y$ effective). The proof of these results use a description of the minimal log discrepancies in terms of dimensions of certain cylinders of the jet spaces of $X$, analogous to the one for the log canonical threshold.

Proposition 5.12. With the notation as above and for $X$ smooth and $Y$ effective,

$$
\begin{aligned}
\operatorname{mld}(W & ; X, Y) \geq \tau \\
& \Longleftrightarrow \\
\operatorname{codim}_{\mathcal{J}_{\infty}(X)}\left(\operatorname{Cont}_{Y}^{\nu} \cap \pi_{0}^{-1}(W)\right) & \geq \sum q_{i} \nu_{i}+\tau \quad \text { for all multi-indices } \nu .
\end{aligned}
$$

where $\operatorname{Cont}_{Y}^{\nu}=\cap_{i} \operatorname{ord}_{Y_{i}}^{-1}\left(\nu_{i}\right)$.

The proof of this is not more than a technical complication of the proof of the log canonical threshold formula we gave above. With this characterization of $\operatorname{mld}(W ; X, Y)$ the proof of inversion of adjunction becomes a matter of determining the co-dimensions of the cylinders involve. In the case that $X$ and $D$ are both smooth this is quite easy (and could be done as an exercise). In general ( $X$ a complete intersection) the combinatorics involved can become quite intricate, $c f$. [16]. 
One should point out that after these results on inversion of adjunction were obtained, Takagi [43] found an alternative approach using positive characteristic methods.

5.5. Geometry of arc spaces without explicit motivic integration. As it should have become apparent by now, the applications of motivic integration to birational geometry are by means of describing certain properties of a variety $X$ in terms of (mostly simpler) properties of its jet spaces $\mathcal{J}_{m}(X)$. Motivic integration serves as the path to make this connection. However, due to some combinatiorial difficulties one encounters along this path, one can ask if there is a more direct relationship. This is indeed the case and it is the content of the paper of [14] of Ein, Lazarsfeld and Mustaţă which is the source of the material in this section.

Their point is that instead of using the birational transformation rule to control the dimension of components of the jet spaces one uses the Key Theorem 3.3 of its proof to more directly get to the desired information.

We keep the notation of a subvariety $Y \subseteq X$ of a smooth variety $X$ and define.

Definition 5.13. Let $Y$ be a subvariety of $X$ and $p \geq 0$ an integer define the contact locus to be the cylinder

$$
\operatorname{Cont}_{Y}^{p} \stackrel{\text { def }}{=} \operatorname{ord}_{Y}^{-1}(p) \subseteq \mathcal{J}_{\infty}(X)
$$

The aim is to understand the components (or at least the dimension) of the cylinders Cont $_{Y}^{p}$ in terms of the data coming from a log resolution of the pair $(X, Y)$. Using the notation of Section 5.1 we fix a log resolution $f: X^{\prime} \rightarrow X$ of the pair $(X, Y)$ and denote $f^{-1} Y=\sum_{1}^{k} a_{i} E_{i}$ and $K_{X^{\prime} / X}=\sum_{1}^{k} b_{i} E_{i}$ where the support of $\sum E_{i}$ is a simple normal crossing divisor.

Definition 5.14. Given $E=\sum_{1}^{k} E_{i}$, a simple normal crossing divisor of $X^{\prime}$, and a multi-index $\nu=\left(\nu_{1}, \ldots, \nu_{k}\right)$ define the multi contact locus

$$
\operatorname{Cont}_{E}^{\nu} \stackrel{\text { def }}{=}\left\{\gamma^{\prime} \in \mathcal{J}_{\infty}\left(X^{\prime}\right) \mid \operatorname{ord}_{E_{i}}\left(\gamma^{\prime}\right)=\nu_{i} \text { for } i=1 \ldots k\right\} .
$$

Definition 5.15. For every cylinder $C \subseteq \mathcal{J}_{\infty}(X)$ there is a well defined notion of codimension, namely

$$
\operatorname{codim} C \stackrel{\text { def }}{=} \operatorname{codim}\left(\mathcal{J}_{m}(X), \pi_{m} C\right)
$$

for $m \gg 0$.

Of course one must check that this is independent of the chosen $m \gg 0$. This however is immediately clear from the definition of cylinder.

Exercise 5.2. For $C \subseteq \mathcal{J}_{\infty}(X)$ a cylinder, show that $\operatorname{codim} C=\operatorname{dim} X-\operatorname{dim} \mu_{X}(C)$.

The following proposition replaces in this new setup the computation of the motivic integral of a normal crossing divisor in Proposition 2.6. Note the comparative simplicity! 
Proposition 5.16. For $E=\sum_{1}^{k} E_{i}$ a simple normal crossing divisor Cont $_{E}^{\nu}$ is a smooth irreducible cylinder of codimension

$$
\operatorname{codim}_{\operatorname{Cont}}^{\nu}=\sum_{1}^{k} \nu_{i}
$$

provided Cont $_{E}^{\nu}$ is nonempty.

Proof. This is a computation in local coordinates. Assume $E$ is locally given by the vanishing of the first $k$ of the coordinates $x_{1}=\ldots=x_{k}=0$. Then, for an arc $\gamma$, which is determined by $\gamma\left(x_{i}\right)=\sum \gamma_{i}^{(j)} t^{j}$ for $i=1 \ldots n$, to have the prescribed contact order with the $E_{i}$ means precisely that $\gamma_{i}^{(j)}=0$ for $j<\nu_{i}$, and $\gamma_{i}^{\left(\nu_{i}\right)} \neq 0$. Hence for $m \gg 0$ we have

$$
\pi_{m}\left(\mathrm{Cont}_{E}^{\nu}\right) \cong\left(\mathbb{A}^{1}-\{0\}\right)^{n} \times \prod \mathbb{A}^{m-\nu_{i}}
$$

and therefore $\operatorname{Cont}_{E}^{\nu}$ is smooth irreducible and of codimension $\sum \nu_{i}$.

The central result (replacing the transformation rule) is the following Theorem

Theorem 5.17. With the notation as above one has for all $p>0$ a finite partition

$$
\operatorname{Cont}_{Y}^{p}=\bigsqcup_{v} f_{\infty} \text { Cont }_{E}^{n}
$$

where the disjoint union is over all multi-indices $\nu$ such that $\sum \nu_{i} a_{i}=p$.

For every multi-index is the set $f_{\infty}$ Cont $_{E}^{v}$ an irreducible cylinder of codimension

$$
\sum \nu_{i}\left(b_{i}+1\right)
$$

In particular, for each irreducible component $Z$ of $\operatorname{Cont}_{Y}^{p}$ there is a unique multiindex $\nu$ such that Cont $_{E}^{\nu}$ dominates $Z$.

Proof. As in the proof of the transformation rule the key ingredient is Theorem 3.3. With this at hand the proof is not difficult.

The condition $\sum \nu_{i} a_{i}=p$ ensures that $f_{\infty} \operatorname{Cont}_{E}^{\nu} \subseteq \operatorname{Cont}_{Y}^{p}$. The surjectivity of $f_{\infty}$ ( $c f$. Exercise 3.2) on the other hand implies the reverse inclusion. The disjoined-ness of the union follows from the fact that there is a one-to-one map between

$$
\mathcal{J}_{\infty}\left(X^{\prime}\right)-\mathcal{J}_{\infty}(E) \stackrel{1-1}{\longrightarrow} \mathcal{J}_{\infty}(X)-\mathcal{J}_{\infty}(Y)
$$

(which is an implication of the valuative criterion for properness as explained in Section 3.0.1) and the observation that each Cont $_{E}^{\nu}$ is contained in the left hand side.

Since Cont $\operatorname{Cont}_{E}^{\nu} \operatorname{Con}_{K_{X^{\prime} / X}}^{\sum b_{i} v_{i}}=\operatorname{ord}_{K_{X^{\prime} / X}}^{-1}$ it follows from Theorem 3.3 (a) and Proposition 3.2 that $f_{\infty} \operatorname{Cont}_{E}^{\nu}$ is a cylinder. Part (b) of Theorem 3.3 shows that

$$
f_{m}: \operatorname{Cont}_{E}^{\nu} \rightarrow f_{m} \operatorname{Cont}_{E}^{\nu}
$$

is a piecewise trivial $\mathbb{A}^{\sum b_{i} \nu_{i}}$-fibration. By Exercise 5.2 is the codimension of Cont $_{E}^{\nu}$ equal to $\sum \nu_{i}$. Hence the codimension of its image under $f_{\infty}$ is

$$
\operatorname{codim}\left(f_{\infty} \operatorname{Cont}_{E}^{\nu}\right)=\sum \nu_{i}+\sum \nu_{i} b_{i}=\sum \nu_{i}\left(b_{i}+1\right)
$$

as claimed. 
As an illustration of this result we recover a very clean proof of the log canonical threshold formula of Theorem 5.1.

Proof of Theorem 5.1. Let $V_{m}$ be an irreducible component of $\mathcal{J}_{m}(Y)$. For some $p \geq$ $m+1$ the set $\operatorname{Cont}_{Y}^{p} \cap\left(\pi_{m}^{X}\right)^{-1} V_{m}$ is open in $\left(\pi_{m}^{X}\right)^{-1} V_{m}$, namely for the smallest $p$ (automatically $\geq m+1$ since each arc in $V_{m}$ has contact order $\geq m+1$ with $Y$ ) such that

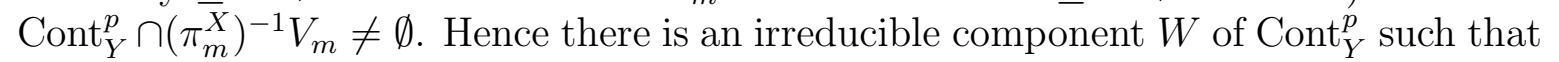
the closure $\bar{W}$ contains $\left(\pi_{m}^{X}\right)^{-1} V_{m}$. By Theorem 5.17 there is a unique multi-index $\nu$ (necessarily $\sum \nu_{i} b_{i}=p$ ) such that $\operatorname{Cont}_{E}^{\nu}$ dominates $W$.

By definition of the log canonical threshold we have $b_{i}+1 \geq \operatorname{lct}(X, Y) a_{i}$ for all $i$ such that we obtain the following inequalities:

$$
\begin{aligned}
\operatorname{codim}\left(V_{m}, \mathcal{J}_{m}(X)\right) & \geq \operatorname{codim} W \\
& =\operatorname{codim} f_{\infty} \operatorname{Cont}_{E}^{\nu} \\
& \geq \sum \nu_{i}\left(b_{i}+1\right) \\
& \geq \sum \nu_{i} \operatorname{lct}(X, Y) a_{i} \\
& =\operatorname{lct}(X, Y) \cdot p=\operatorname{lct}(X, Y) \cdot(m+1)
\end{aligned}
$$

As this holds for every irreducible component of $\mathcal{J}_{m}(Y)$ we get

$$
\operatorname{lct}(X, Y) \leq \frac{\operatorname{codim}\left(\mathcal{J}_{m}(Y), \mathcal{J}_{m}(X)\right)}{m+1}
$$

To see that there is equality for some $m$ we pick an index $i$ such that $\operatorname{lct}(X, Y)=\frac{b_{i}+1}{a_{i}}$ and $m+1$ divisible by $a_{i}$. Let $\nu$ be the multi-index which is zero everywhere except at the $i$ th spot, where it is $\frac{m+1}{a_{i}}$. Then $f_{\infty} \operatorname{Cont}_{E}^{\nu} \subseteq \operatorname{Cont}_{Y}^{m+1} \subseteq\left(\pi_{m}^{X}\right)^{-1} \mathcal{J}_{m}(Y)$ and by Theorem 5.17 the codimension $f_{\infty} \operatorname{Cont}_{E}^{\nu}$ is equal to $\frac{m+1}{a_{i}}\left(b_{i}+1\right)=\operatorname{lct}(X, Y) \cdot(m+1)$. Hence in particular $\operatorname{codim}\left(\mathcal{J}_{m}(Y), \mathcal{J}_{m}(X)\right) \leq \operatorname{lct}(X, Y) \cdot(m+1)$ for this chosen $m+1$. This finishes the argument.

In summary, the above agument shows that the irreducible components $V$ of $\mathcal{J}_{m}(Y)$ of maximal possible dimension, that is the ones that compute the log canonical theshold as $\operatorname{lct}(X, Y)=\operatorname{codim}\left(\mathcal{J}_{m}(X), V\right)$ are dominated by multi-contact loci $\operatorname{Cont}_{E}^{\nu}$ with $\nu_{i} \neq 0$ for all the indices $i$ such that $E_{i}$ computes the the log canonical theshold (meaning $\left.\operatorname{lct}(X, Y)=\frac{b_{i}+1}{a_{i}}\right)$.

We want to finish these notes with Mustaţă's characterization of rational singularities for complete intersections in terms of arc spaces. This was indeed the first application of motivic integration to characterizing singularities. With the just developed viewpoint this result is not too difficult anymore.

Theorem 5.18. Let $Y \subseteq X$ be a reduced and irreducible locally complete intersection subvariety of codimension $c$. Then the jet spaces $\mathcal{J}_{m}(Y)$ are irreducible for all $m$ if and only if $Y$ has rational singularities.

Proof. Let $f: X^{\prime} \rightarrow X$ be a $\log$ resolution of $(X, Y)$ which dominates the blowup of $X$ along $Y$. Keeping the previous notation we may assume that $E_{1}$ is the exceptional 
divisor of this blowup. In [34] Theorem 2.1 it is shown that $Y$ has at worst rational singularities if and only if $b_{i} \geq c a_{i}$ for every $i \geq 2$. Hence we must show

$$
\mathcal{J}_{m}(Y) \text { is irreducible for all } m \geq 1 \Longleftrightarrow b_{i} \geq c a_{i} \text { for } i \geq 2
$$

Assume that $\mathcal{J}_{m}(Y)$ is not irreducible, that is we have a component $V \subseteq \mathcal{J}_{m}(Y)$ other than the main component $\mathcal{J}_{m}(Y-\operatorname{Sing} Y)$. As in the previous proof we have $W \subseteq$ Cont $_{Y}^{p}$ with $p \geq m+1$ whose closure contains $\pi_{m}^{-1}(V)$. By Theorem 5.17 this component is dominated by some multi-contact locus $\operatorname{Cont}_{E}^{\nu}$ for $\nu \neq(m+1,0, \ldots, 0)$ since the latter is the multi-index corresponding to the multi-contact locus dominating $\pi_{m}^{-1}\left(\mathcal{J}_{m}(Y-\operatorname{Sing} Y)\right)$. Since $Y \subseteq X$ is a local complete intersection of codimension $c$ we have $\operatorname{codim}\left(V, \mathcal{J}_{m}(X)\right) \leq(m+1) \cdot c$. To arrive at a contradiction assume now that $Y$ has rational singularities, that is assume that $b_{i} \geq c a_{i}$ for $i \geq 2$. Then

$$
\begin{aligned}
(m+1) \cdot c & \geq \operatorname{codim}(W) \\
& =\nu_{1} \cdot c \sum_{i \geq 2} \nu_{i}\left(b_{i}+1\right) \\
& \geq c \cdot \sum_{i \geq 1} \nu_{i} a_{i}+\sum_{\geq 2} \nu_{i} \quad\left(\text { since } b_{i} \geq c a_{i}\right) \\
& =c \cdot p+\sum_{i \geq 2} \nu_{i} \geq c \cdot(m+1)+\sum_{i \geq 2} \nu_{i}
\end{aligned}
$$

Hence for $i \geq 2$ we must have $\nu_{i}=0$, a contradiction.

Conversely, suppose $b_{i}<c \cdot a_{i}$ for some $i \geq 2$. Setting $v$ to be the multi-index with all entries zero except the $i$ th equal to 1 . Let $(m+1)=a_{i}$, then the $\operatorname{Cont}_{E}^{\nu}$ maps to an irreducible subset $W \subseteq \operatorname{Cont}_{Y}^{m+1}$ of codimension $(m+1) \cdot c$. Hence $\pi_{m+1}(W)$ is an irreducible component of $\mathcal{J}_{m}(Y)$ of codimension $(m+1) \cdot c$ which is not the component $\overline{\mathcal{J}_{m}(Y-\operatorname{Sing} Y)}$. Hence $\mathcal{J}_{m}(Y)$ is not irreducible.

\section{Appendix A. An elementary proof of the Transformation RUle.}

We present Looijenga's [33] elementary proof of Theorem 3.3 which then leads to a proof of the transformation formula avoiding weak factorization. For this we have to investigate more carefully the definition of the relative canonical divisor $K_{X^{\prime} / X}$ and suitably interpret the contact multiplicity of an arc $\gamma$ with $K_{X / X^{\prime}}$.

A.1. The relative canonical divisor and differentials. Let us consider the first fundamental exact sequence for Kähler differentials, as it plays a pivotal role in all that follows.

The morphism $f: X^{\prime} \longrightarrow X$ induces a linear map, its derivative, $f^{*} \Omega_{X} \stackrel{d f}{\longrightarrow} \Omega_{X^{\prime}}$ which is part of the first fundamental exact sequence for Kähler differentials:

$$
0 \longrightarrow f^{*} \Omega_{X} \stackrel{d f}{\longrightarrow} \Omega_{X^{\prime}} \longrightarrow \Omega_{X^{\prime} / X} \longrightarrow 0
$$

Note that by our assumption of smoothness, the $\mathcal{O}_{X^{\prime}}$-modules $f^{*} \Omega_{X}$ and $\Omega_{X^{\prime}}$ are locally free of rank $n=\operatorname{dim} X$. Since, by birationality of $f, \Omega_{X^{\prime} / X}$ has rank zero, the 
first map is injective as well. Taking the $n$th exterior power we obtain the map

$$
0 \longrightarrow f^{*} \Omega_{X}^{n} \stackrel{\wedge^{n} d f}{\longrightarrow} \Omega_{X^{\prime}}^{n}
$$

of locally free $\mathcal{O}_{X^{\prime}}$ modules of rank 1 . If we set $\omega=\Omega^{n}$ and tensor the above sequence with the invertible sheaf $\omega_{X^{\prime}}^{-1}$ we obtain

$$
f^{*} \omega_{X} \otimes \omega_{X^{\prime}}^{-1} \subseteq \mathcal{O}_{X^{\prime}}
$$

thus identifying $f^{*} \omega_{X} \otimes \omega_{X^{\prime}}^{-1}$ with a locally principal ideal in $\mathcal{O}_{X^{\prime}}$, which we shall denote by $J_{X^{\prime} / X}$ (so, by definition, $J_{X^{\prime} / X}$ is the 0 -th Fitting ideal of $\Omega_{X^{\prime} / X}$ ). Now define $K_{X^{\prime} / X}$ to be the Cartier divisor which is locally given by the vanishing of $J_{X^{\prime} / X}$. It is important to note that $K_{X^{\prime} / X}$ is defined as an effective divisor and not just as a divisor class. By choosing bases for the free $\mathcal{O}_{X^{\prime}}$-modules $f^{*} \Omega_{X}$ and $\Omega_{X^{\prime}}$ the map $d f$ is given by a $n \times n$ matrix with entries in $\mathcal{O}_{X^{\prime}}$. Its determinant is a local defining equation for $K_{X^{\prime} / X}$.

Let $L$ be an extension field of $k$ and let $\gamma:$ Spec $L \llbracket t \rrbracket \rightarrow X$ be a $L$-rational point of $\mathcal{J}_{\infty}\left(X^{\prime}\right)$, and assume that $\operatorname{ord}_{K_{X^{\prime} / X}}(\gamma)=e$. By definition of contact order, this means that $\left(t^{e}\right)=\gamma^{*}\left(J_{X^{\prime} / X}\right) \subseteq L \llbracket t \rrbracket$. As $J_{X^{\prime} / X}$ is locally generated by $\operatorname{det} d f \in \mathcal{O}_{X^{\prime}}$ (well defined up to unit) we obtain that $\left(t^{e}\right)=\operatorname{det}\left(\gamma^{*}(d f)\right)$. The pullback of the sequence (8) along $\gamma$ illustrates the situation:

$$
0 \longrightarrow(f \circ \gamma)^{*} \Omega_{X} \stackrel{\gamma^{*} d f}{\longrightarrow} \gamma^{*} \Omega_{X^{\prime}} \longrightarrow \gamma^{*} \Omega_{X^{\prime} / X} \longrightarrow 0
$$

Since $L \llbracket t \rrbracket$ is a PID, we can choose bases of $(f \circ \gamma)^{*} \Omega_{X}$ and $\gamma^{*} \Omega_{X^{\prime}}$ such that $\gamma^{*}(d f)$, a map of free $L \llbracket t \rrbracket$ modules of rank $n$, is given by a diagonal matrix. With respect to this basis the exact sequence (8) takes the form

$$
0 \rightarrow L \llbracket t \rrbracket^{n} \stackrel{\left(\begin{array}{ccc}
t^{e_{1}} & & 0 \\
& \ddots & \\
0 & & t^{e_{n}}
\end{array}\right)}{\longrightarrow} L \llbracket t \rrbracket^{n} \rightarrow \oplus \frac{L \llbracket t \rrbracket}{\left(t^{e_{i}}\right)} \longrightarrow 0
$$

The condition that $\operatorname{ord}_{K_{X^{\prime} / X}}(\gamma)=e$ translates into $\sum_{i=1}^{n} e_{i}=e$ or, equivalently, into saying that the rightmost module is torsion of length $e$.

A.2. Proof of Theorem 3.3. We start by recalling the statement of Theorem 3.3 we want to proof slightly reformulated in order to set up the notation that is used in its proof below.

Theorem A.1. Let $f: X^{\prime} \longrightarrow X$ be a proper birational morphism of smooth $k$-varieties. Let $C_{e}^{\prime}=\operatorname{ord}_{K_{X^{\prime} / X}}^{-1}(e)$ where $K_{X^{\prime} / X}$ is the relative canonical divisor and let $C_{e} \stackrel{\text { def }}{=}$ $f_{\infty} C_{e}^{\prime}$. Let $\gamma \in C_{e}^{\prime}$ an L-point of $\mathcal{J}_{\infty}\left(X^{\prime}\right)$, that is a map $\gamma^{*}: \mathcal{O}_{X^{\prime}} \longrightarrow L \llbracket t \rrbracket$, satisfying $\gamma^{*}\left(J_{X^{\prime} / X}\right)=\left(t^{e}\right)$, with $L \supseteq k$ a field extension. Then for $m \geq 2 e$ one has:

(a') For all $\xi \in \mathcal{J}_{\infty}(X)$ such that $\pi_{m}^{X}(\xi)=f_{m}\left(\pi_{m}^{X^{\prime}}(\gamma)\right)$ there is $\gamma^{\prime} \in \mathcal{J}_{\infty}\left(X^{\prime}\right)$ such that $f_{\infty}\left(\gamma^{\prime}\right)=\xi$ and $\pi_{m-e}^{X^{\prime}}\left(\gamma^{\prime}\right)=\pi_{m-e}^{X^{\prime}}(\gamma)$. In particular, the fiber of $f_{m}$ over $f_{m}\left(\gamma_{m}\right)$ lies in the fiber of $\pi_{m-e}^{m}$ over $\gamma_{m-e}$.

(a) $\pi_{m}\left(C_{e}^{\prime}\right)$ is a union of fibers of $f_{m}$.

(b) The map $f_{m}: \pi_{m}^{X^{\prime}}\left(C_{e}^{\prime}\right) \longrightarrow C_{e}$ is a piecewise trivial $\mathbb{A}^{e}$ fibration. 
Proof. To ease notation we will denote truncation by lower index, i.e. write $\gamma_{m}$ as shorthand for $\pi_{m}^{X}(\gamma)$. We already pointed out before that (a') implies (a):

The proof of (b) can be divided into two steps. First we show that the fiber of $f_{m}$ over $f_{m}\left(\gamma_{m}\right)$ can be naturally identified with $\operatorname{Der}_{\mathcal{O}_{X}}\left(\mathcal{O}_{X^{\prime}}, \frac{L \llbracket t \rrbracket}{\left(t^{m+1}\right)}\right)$. Then we have to show that the latter is an affine space of dimension $e$. As this is easy let's do it first: Immediately preceding this proposition we noted that the cokernel of $\gamma^{*}(d f)$ is torsion of length $e$ as a $L \llbracket t \rrbracket$-module. This cokernel is $\gamma^{*} \Omega_{X^{\prime} / X}$. Since $m>e$ the dual, $\operatorname{Hom}_{L \llbracket t \rrbracket}\left(\gamma^{*} \Omega_{X^{\prime} / X}, \frac{L \llbracket t \rrbracket}{\left(t^{m+1}\right)}\right)$, is also torsion of length $e$. Using adjointness of $\gamma^{*}$ and $\gamma_{*}$ this Hom is just $\operatorname{Hom}_{\mathcal{O}_{X^{\prime}}}\left(\Omega_{X^{\prime} / X}, \gamma_{*} \frac{L \llbracket t \rrbracket}{\left(t^{m+1}\right)}\right)$, which is equal to $\operatorname{Der}_{\mathcal{O}_{X}}\left(\mathcal{O}_{X^{\prime}}, \gamma_{*} \frac{L \llbracket t \rrbracket}{\left(t^{m+1}\right)}\right)$ essentially by definition of $\Omega_{X^{\prime} / X}$. This shows that $\operatorname{Der}_{\mathcal{O}_{X}}\left(\mathcal{O}_{X^{\prime}}, \gamma_{*} \frac{L \llbracket t \rrbracket}{\left(t^{m+1}\right)}\right)$ is isomorphic to $\mathbb{A}_{L}^{e}$. Thus we are left to show the identification $(\diamond \diamond \diamond)$ of the following diagram the last line of which is the first exact sequence for derivations, analogous to the above exact sequence of Kähler differentials.

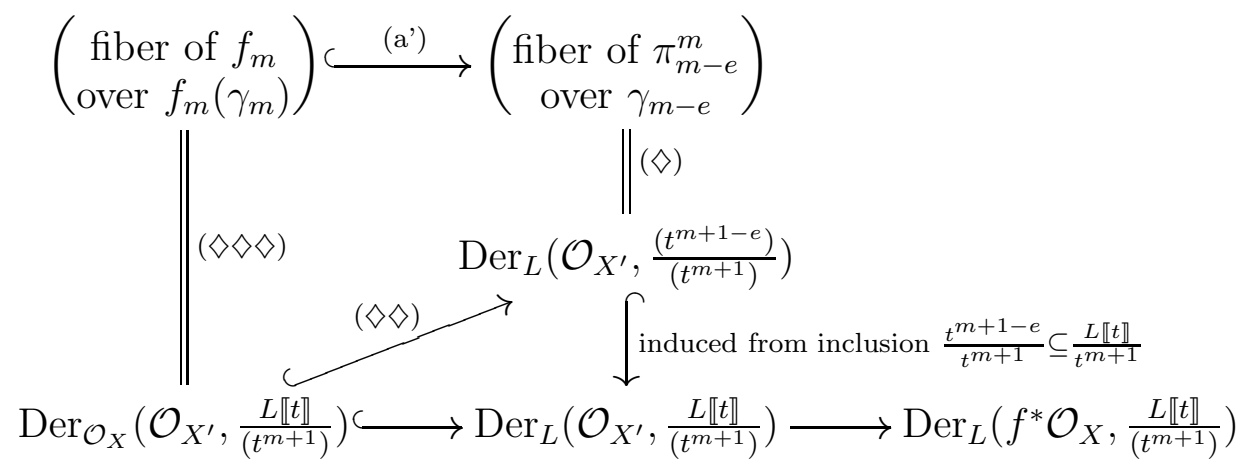

The identification $(\diamond)$ is given by sending $\gamma_{m}^{\prime}$ to $\gamma_{m}^{\prime}-\gamma_{m}$ which, since $m \geq 2 e$, can easily $^{41}$ be checked to define an $L$-derivation $\mathcal{O}_{X^{\prime}} \rightarrow \frac{\left(t^{m+1-e}\right)}{\left(t^{m+1}\right)}$. In this way (and using $\left.\left(\mathrm{a}^{\prime}\right)\right)$ we think of $f_{m}^{-1}\left(f_{m}\left(\gamma_{m}\right)\right)$ as a subspace of $\operatorname{Der}_{L}\left(\mathcal{O}_{X^{\prime}}, \frac{\left(t^{m+1-e}\right)}{\left(t^{m+1}\right)}\right)$. As this is the $t^{e}-$ torsion part of $\operatorname{Der}_{L}\left(\mathcal{O}_{X^{\prime}}, \frac{L \llbracket t \rrbracket}{\left(t^{m+1}\right)}\right)$ and since we just observed that $\operatorname{Der}_{\mathcal{O}_{X}}\left(\mathcal{O}_{X^{\prime}}, \frac{L \llbracket t \rrbracket}{\left(t^{m+1}\right)}\right)$

\footnotetext{
${ }^{41}$ Fix an homomorphism $\gamma: R \longrightarrow S$ of $k$-algebras which makes $S$ into an $R$-algebra. For any ideal $I \in S$ with $I^{2}=0$ one has a map

$$
\left\{\gamma^{\prime} \in \operatorname{Hom}_{k-\operatorname{alg}}(R, S) \mid \operatorname{Im}\left(\gamma^{\prime}-\gamma\right) \subseteq I\right\} \longrightarrow \operatorname{Der}_{k}(R, I)
$$

by sending $\gamma^{\prime}$ to $\gamma^{\prime}-\gamma$. To check that $\left(\gamma^{\prime}-\gamma\right)$ is indeed a derivation one has to make the following calculation verifying the Leibniz rule (note that the $R$ algebra structure on $S$ is via $\gamma$ ):

$$
\begin{aligned}
\left(\gamma^{\prime}-\gamma\right)(x y) & -\left(\left(\gamma^{\prime}-\gamma\right)(x) \gamma(y)+\gamma(x)\left(\gamma^{\prime}-\gamma\right)(y)\right) \\
& =\gamma^{\prime}(x) \gamma^{\prime}(y)-\gamma(x) \gamma(y)-\gamma^{\prime}(x) \gamma(y)+\gamma(x) \gamma(y)-\gamma(x) \gamma^{\prime}(y)+\gamma(x) \gamma(y) \\
& =\gamma^{\prime}(x)\left(\gamma^{\prime}(y)-\gamma(y)\right)-\gamma(x)\left(\gamma^{\prime}(y)-\gamma(y)\right) \\
& =\left(\gamma^{\prime}-\gamma\right)(x) \cdot\left(\gamma^{\prime}-\gamma\right)(y)=0
\end{aligned}
$$
}

The last line is zero by the assumption that $\operatorname{Im}\left(\gamma^{\prime}-\gamma\right) \subseteq I$ and $I^{2}=0$. The obvious inverse map sending a derivation $\delta$ to $\gamma+\delta$ shows that the two sets in (11) are equal. This setup clearly applies in our situation: $R=\mathcal{O}_{X^{\prime}}, S=L \llbracket t \rrbracket / t^{m+1}, I=\left(t^{m+1-e}\right)$ where $m \geq 2 e$ ensures that $I^{2}=0$. 
is torsion of lenght $e$ the inclusion $(\diamond \diamond)$ is also clear, and thus $(\diamond \diamond \diamond)$ becomes a statement about subsets of $\operatorname{Der}_{L}\left(\mathcal{O}_{X^{\prime}}, \frac{\left(t^{m+1-e}\right)}{\left(t^{m+1}\right)}\right)$.

Let $\left(\gamma_{m}^{\prime}-\gamma_{m}\right) \in \operatorname{Der}_{L}\left(\mathcal{O}_{X^{\prime}}, \frac{\left(t^{m+1-e}\right)}{\left(t^{m+1}\right)}\right)$. The image of $\left(\gamma_{m}^{\prime}-\gamma_{m}\right)$ in $\operatorname{Der}_{L}\left(f^{*} \mathcal{O}_{X}, \frac{L \llbracket t \rrbracket}{\left(t^{m+1}\right)}\right)$ is $\gamma_{m}^{\prime} \circ f-\gamma_{m} \circ f$. This is zero (i.e. $\gamma_{m}^{\prime} \in \operatorname{Der}_{\mathcal{O}_{X}}\left(\mathcal{O}_{X^{\prime}}, \frac{L \llbracket t \rrbracket}{\left(t^{m+1}\right)}\right)$ ) if and only if $f_{m}\left(\gamma_{m}^{\prime}\right)=$ $f_{m}\left(\gamma_{m}\right)$, that is if and only if $\gamma_{m}^{\prime}$ is in the fiber of $f_{m}$ over $f_{m}\left(\gamma_{m}\right)$. This concludes the proof of (b).

In order to come by the element $\gamma^{\prime} \in \mathcal{J}_{\infty}\left(X^{\prime}\right)$ as claimed in (a') we construct a sequence of $\operatorname{arcs} \gamma^{k} \in \mathcal{J}_{\infty}\left(X^{\prime}\right)$ satisfying the following two properties for all $k \geq m$ :

(1) $\pi_{k}\left(f_{\infty}\left(\gamma^{k}\right)\right)=\pi_{k}(\xi)$ and

(2) $\pi_{k-1-e}\left(\gamma^{k}\right)=\pi_{k-1-e}\left(\gamma^{k-1}\right)$ and $\pi_{m-e}\left(\gamma^{k}\right)=\pi_{m-e}(\gamma)$.

Clearly, setting $\gamma^{m-1}=\gamma^{m}=\gamma$ these conditions hold for $k=m$. Furthermore, the second condition implies that the limit $\gamma^{\prime} \stackrel{\text { def }}{=} \lim _{k} \gamma^{k}$ exists and that $\pi_{m-e}\left(\gamma^{\prime}\right)=\pi_{m-e}(\gamma)$. The first condition shows that $f_{\infty}\left(\gamma^{\prime}\right)=\xi$. Thus we are left with constructing the sequence $\gamma^{k}$. This is done inductively. As we already verified the solution for $k=m$ we assume to have $\gamma^{k}$ and $\gamma^{k-1}$ as claimed - now $\gamma^{k+1}$ is constructed as follows:

Since $\pi_{k}\left(f\left(\gamma^{k}\right)\right)=\pi_{k}(\xi)$ we can view their difference as a derivation $\delta=\xi-f \circ \gamma^{k} \in$ $\operatorname{Der}_{L}\left(\mathcal{O}_{X}, \frac{\left(t^{k+1}\right)}{\left(t^{k+2}\right)}\right)$ which we identify with $\operatorname{Hom}_{L \llbracket t \rrbracket}\left(\gamma^{k *} f^{*} \Omega_{X}, \frac{\left(t^{k+1}\right)}{\left(t^{k+2}\right)}\right)$. The latter module appears in $\operatorname{Hom}_{L \llbracket t \rrbracket}\left(\ldots, \frac{L \llbracket t \rrbracket \rrbracket}{\left(t^{k+2}\right)}\right)$ applied to the sequence (9), where $\gamma^{k}$ takes the place of $\gamma:$

$$
\begin{aligned}
\operatorname{Hom}\left(\gamma^{k *} \Omega_{X / X^{\prime}}, \frac{L \llbracket t \rrbracket}{\left(t^{k+2}\right)}\right) & \longrightarrow \operatorname{Hom}\left(\gamma^{k *} \Omega_{X^{\prime}}, \frac{L \llbracket t \rrbracket}{\left(t^{k+2}\right)}\right) \stackrel{d f}{\longrightarrow} \operatorname{Hom}\left(\gamma^{k *} f^{*} \Omega_{X}, \frac{L \llbracket t \rrbracket}{\left(t^{k+2}\right)}\right) \\
\cup & \cup \\
& \operatorname{Hom}\left(\gamma^{k *} \Omega_{X^{\prime}}, \frac{\left(t^{k+1-e}\right)}{\left(t^{k+2}\right)}\right) \ni \delta^{\prime} \mapsto \delta \in \operatorname{Hom}\left(\gamma^{k *} f^{*} \Omega_{X}, \frac{\left(t^{k+1}\right)}{\left(t^{k+2}\right)}\right)
\end{aligned}
$$

In order to understand this better we turn to the same sequence, but with respect to the basis as in sequence (10), where it takes this form:

$$
\begin{aligned}
& \oplus \frac{\left(t^{k+1-e_{i}}\right)}{\left(t^{k+2}\right)} \longrightarrow\left(\frac{L \llbracket t \rrbracket}{\left(t^{k+2}\right)}\right)^{n} \stackrel{\left(\begin{array}{ccc}
t^{e_{1}} & & 0 \\
& \ddots & \\
0 & & t^{e_{n}}
\end{array}\right)}{\longrightarrow}\left(\frac{L \llbracket t]}{\left(t^{k+2}\right)}\right)^{n} \\
& \cup \quad U \\
& \left(\frac{\left(t^{k+1-e}\right)}{\left(t^{k+2}\right)}\right)^{n} \quad \delta \in\left(\frac{t^{k+1}}{\left(t^{k+2}\right)}\right)^{n}
\end{aligned}
$$

Now it becomes clear that $\delta$ lies in the image of $d f$ since $e$ and therefore all $e_{i}$ are less than $m+1 \leq k+1$. Furthermore, any pre-image $\delta^{\prime}$ must lie in $\operatorname{Hom}\left(\gamma^{k *} \Omega_{X^{\prime}}, \frac{\left(t^{k+1-e}\right)}{\left(t^{k+2}\right)}\right)$ by the shape of the matrix and the fact that $e_{i} \leq e$ for all $e$. Now pick any such preimage $\delta^{\prime}$ and define $\gamma^{k+1} \stackrel{\text { def }}{=} \delta^{\prime}+\gamma^{k}$. This is an arc in $X^{\prime}$ with $\pi_{k-e}\left(\gamma^{k+1}\right)=\pi_{k-e}\left(\gamma^{k}\right)$. Furthermore since $d f\left(\delta^{\prime}\right)=\delta$ we get

$$
\gamma^{k+1} \circ f-\gamma^{k} \circ f=\delta=\xi-\gamma^{k} \circ f \bmod \left(t^{k+2}\right)
$$

and thus $\pi_{k+1}\left(\gamma^{k+1} \circ f\right)=\pi_{k+1}\left(f_{\infty}\left(\gamma^{k+1}\right)\right)=\pi_{k+1}(\xi)$. 
With this proof of Theorem 3.3 at hand a proof of the Transformation rule follows immediately as indicated in Section 3.

\section{REFERENCES}

[1] Dan Abramovich, Kalle Karu, Kenji Matsuki, and Jarosław Włodarczyk, Torification and factorization of birational maps, J. Amer. Math. Soc. 15 (2002), no. 3, 531-572 (electronic).

[2] Victor V. Batyrev, Stringy Hodge numbers of varieties with Gorenstein canonical singularities, Integrable systems and algebraic geometry (Kobe/Kyoto, 1997), World Sci. Publishing, River Edge, NJ, 1998, pp. 1-32.

[3] Victor V. Batyrev and Lev A. Borisov, Mirror duality and string-theoretic Hodge numbers, Invent. Math. 126 (1996), no. 1, 183-203.

[4] Franziska Bittner, The universal Euler characteristic for varieties of characteristic zero, Compos. Math. 140 (2004), no. 4, 1011-1032.

[5] Siegfried Bosch, Werner Lütkebohmert, and Michel Raynaud, Néron models, Ergebnisse der Mathematik und ihrer Grenzgebiete (3), vol. 21, Springer-Verlag, Berlin, 1990.

[6] R. Cluckers and F. Loeser, Constructible motivic functions and motivic integration, preprint, 2003.

[7] Alastair Craw, An introduction to motivic integration, Strings and geometry, Clay Math. Proc., vol. 3, Amer. Math. Soc., Providence, RI, 2004, pp. 203-225.

[8] Pierre Deligne, Théorie de Hodge. I, Actes du Congrès International des Mathématiciens (Nice, 1970), Tome 1, Gauthier-Villars, Paris, 1971, pp. 425-430.

[9] _ Théorie de Hodge. II, Inst. Hautes Études Sci. Publ. Math. (1971), no. 40, 5-57.

[10] _ Théorie de Hodge. III, Inst. Hautes Études Sci. Publ. Math. (1974), no. 44, 5-77.

[11] J. Denef and F. Loeser, Geometry on arc spaces of algebraic varieties, arXiv:math.AG/0006050.

$[12] \_$, Germs of arcs on singular algebraic varieties and motivic integration, Invent. Math. 135 (1999), 201-232, arXiv:math.AG/9803039.

[13] Jan Denef and Francois Loeser, Geometry on Arc Spaces, Notes of a talk available at http://www.wis.kuleuven.ac.be/algebra/NotesCambridge/.

[14] Lawrence Ein, Robert Lazarsfeld, and Mircea Mustaţă, Contact loci in arc spaces, Compos. Math. 140 (2004), no. 5, 1229-1244.

[15] Lawrence Ein and Mircea Mustata, The log canonical threshold of homogeneous affine hypersurfaces, arXiv:math.AG/0105113.

[16] Lawrence Ein and Mircea Mustaţă, Inversion of adjunction for local complete intersection varieties, Amer. J. Math. 126 (2004), no. 6, 1355-1365.

[17] Lawrence Ein, Mircea Mustaţă, and Takehiko Yasuda, Jet schemes, log discrepancies and inversion of adjunction, Invent. Math. 153 (2003), no. 3, 519-535.

[18] David Eisenbud, Commutative algebra, Springer-Verlag, New York, 1995.

[19] Marvin J. Greenberg, Schemata over local rings, Ann. of Math. (2) 73 (1961), 624-648.

$[20]$ _ Schemata over local rings. II, Ann. of Math. (2) 78 (1963), 256-266.

[21] _ Rational points in Henselian discrete valuation rings, Inst. Hautes Études Sci. Publ. Math. (1966), no. 31, 59-64.

[22] A. Grothendieck, Éléments de géométrie algébrique. I. Le langage des schémas, Inst. Hautes Études Sci. Publ. Math. (1960), no. 4, 228.

[23] _ Éléments de géométrie algébrique. IV. Étude locale des schémas et des morphismes de schémas IV, Inst. Hautes Études Sci. Publ. Math. (1967), no. 32, 361.

[24] Thomas C. Hales, Can p-adic integrals be computed?, Contributions to automorphic forms, geometry, and number theory, Johns Hopkins Univ. Press, Baltimore, MD, 2004, pp. 313-329.

[25] — What is motivic measure?, Bull. Amer. Math. Soc. (N.S.) 42 (2005), no. 2, 119-135 (electronic).

[26] Robin Hartshorne, Algebraic geometry, Springer, New York, 1973. 
[27] Shihoko Ishii and János Kollár, The Nash problem on arc families of singularities, Duke Math. J. 120 (2003), no. 3, 601-620.

[28] Tetsushi Ito, Stringy Hodge numbers and p-adic Hodge theory, Compos. Math. 140 (2004), no. 6, $1499-1517$.

[29] János Kollár, Singularities of pairs, Algebraic geometry-Santa Cruz 1995, Proc. Sympos. Pure Math., vol. 62, Amer. Math. Soc., Providence, RI, 1997, pp. 221-287.

[30] János Kollár and Shigefumi Mori, Birational geometry of algebraic varieties, Cambridge Tracts in Mathematics, vol. 134, Cambridge University Press, Cambridge, 1998, With the collaboration of C. H. Clemens and A. Corti, Translated from the 1998 Japanese original.

[31] M. Kontsevich, Lecture at Orsay, 1995.

[32] Robert Lazarsfeld, Positivity in algebraic geometry. II, Ergebnisse der Mathematik und ihrer Grenzgebiete. 3. Folge. A Series of Modern Surveys in Mathematics [Results in Mathematics and Related Areas. 3rd Series. A Series of Modern Surveys in Mathematics], vol. 49, Springer-Verlag, Berlin, 2004, Positivity for vector bundles, and multiplier ideals.

[33] Eduard Looijenga, Motivic measures, Astérisque (2002), no. 276, 267-297, Séminaire Bourbaki, Vol. 1999/2000.

[34] Mircea Mustata, Jet Schemes of Locally Complete Intersection Canonical Singularities, arXiv:math.AG/0008002.

[35] _ Jet Schemes of Locally Complete Intersection Canonical Singularities, Invent. Math. 145 (2001), 397-424, arXiv:math.AG/0008002.

[36] _ Singularities of Pairs via Jet Schemes, J. Amer. Math. Soc. 15 (2002), 599-615, arXiv:math.AG/0102201.

[37] John F. Nash, Jr., Arc structure of singularities, Duke Math. J. 81 (1995), no. 1, 31-38 (1996), A celebration of John F. Nash, Jr.

[38] N. Naumann, Algebraic independence in the Grothendieck ring of varieties, arXiv:math.AG/0403075.

[39] Bjorn Poonen, The Grothendieck ring of varieties is not a domain, Math. Res. Lett. 9 (2002), no. 4, 493-497.

[40] Miles Reid, La correspondance de McKay, Astérisque (2002), no. 276, 53-72, Séminaire Bourbaki, Vol. 1999/2000.

[41] Julien Sebag, Intégration motivique sur les schémas formels, Bull. Soc. Math. France 132 (2004), no. $1,1-54$.

[42] Vyacheslav V. Shokurov, Letters of a bi-rationalist. IV. Geometry of log flips, Algebraic geometry, de Gruyter, Berlin, 2002, pp. 313-328.

[43] Shunsuke Takagi, F-singularities of pairs and Inversion of Adjunction of arbitrary codimension, arXiv:math.AG/0305286.

[44] Willem Veys, Arc spaces, motivic integration and stringy invariants, arXiv:math.AG/0401374.

[45] Takehiko Yasuda, Dimensions of jet schemes of log singularities, Amer. J. Math. 125 (2003), no. $5,1137-1145$.

Universität Duisburg-Essen, Standort Essen, 45117 Essen, Germany

E-mail address: manuel.blickle@uni-essen.de

$U R L:$ www.mabli.org 